\title{
Equivariant prequantization bundles on the space of connections and characteristic classes
}

\author{
Roberto Ferreiro Pérez ${ }^{1}$ (D)
}

Received: 10 March 2017 / Accepted: 16 April 2018 / Published online: 24 April 2018

(C) Fondazione Annali di Matematica Pura ed Applicata and Springer-Verlag GmbH Germany, part of Springer Nature 2018

\begin{abstract}
We show how characteristic classes determine equivariant prequantization bundles over the space of connections on a principal bundle. These bundles are shown to generalize the Chern-Simons line bundles to arbitrary dimensions. Our result applies to arbitrary bundles, and we study the action of both the gauge group and the automorphisms group. The action of the elements in the connected component of the identity of the group generalizes known results in the literature. The action of the elements not connected with the identity is shown to be determined by a characteristic class by using differential characters and equivariant cohomology. We extend our results to the space of Riemannian metrics and the actions of diffeomorphisms. In dimension 2 , a $\Gamma_{M}$-equivariant prequantization bundle of the WeilPetersson symplectic form on the Teichmüller space is obtained, where $\Gamma_{M}$ is the mapping class group of the surface $M$.
\end{abstract}

Keywords Equivariant prequantization bundle - Space of connections - Equivariant characteristic classes · Differential characters · Chern-Simons line bundle

Mathematics Subject Classification Primary 53C05; Secondary 53C08 · 70S15 · 58D27

\section{Introduction}

In this paper, we study the relationship between characteristic classes and equivariant prequantization line bundles over the space of connections. We recall two classical examples of this relation (see Sect. 2 for the notation).

In the first example, let $\Sigma$ be a closed (i.e., compact and without boundary) oriented surface, $P=\Sigma \times S U(2)$ the trivial principal $S U(2)$-bundle, and $p \in I_{\mathbb{Z}}^{2}(S U(2))$ the polynomial

Roberto Ferreiro Pérez

roferreiro@ccee.ucm.es

1 Departamento de Economía Financiera y Contabilidad I, Facultad de Ciencias Económicas y Empresariales, Universidad Complutense de Madrid, Campus de Somosaguas, 28223 Pozuelo de Alarcón, Spain 
associated with the second Chern class. We denote by $\mathcal{A}$ and $\widetilde{\mathcal{A}}$ the spaces of connections and irreducible connections on $P$. In [3], Atiyah and Bott show that this polynomial determines a symplectic structure $\sigma$ on the space of connections $\mathcal{A}$ which is invariant under the action of the group $\mathcal{G}$ of gauge transformations. Moreover, the curvature map determines a moment map $\mu$ for $\sigma$. By symplectic reduction, a symplectic structure $\sigma$ on the moduli space of irreducible flat connections $\widetilde{\mathcal{F}} / \mathcal{G}$ is obtained. Furthermore, in [24] it is shown that the action of $\mathcal{G}$ admits a lift to $\widetilde{\mathcal{A}} \times U(1)$ by $U(1)$-bundle automorphisms, hence defining a $\mathcal{G}$-equivariant $U(1)$ bundle over $\widetilde{\mathcal{A}}$ (or what is equivalent, a $\mathcal{G}$-equivariant Hermitian line bundle). By taking the quotient, they obtain an Hermitian line bundle $\mathcal{L} \rightarrow \widetilde{\mathcal{F}} / \mathcal{G}$ (which is proved to be isomorphic to the Quillen determinant line bundle) and a natural connection on $\mathcal{L}$ whose curvature is $\underline{\sigma}$. We remark that all these constructions can be done based only on the polynomial $p$.

The second example is the classical 3-dimensional Chern-Simons theory. Let $M$ be a compact 3-dimensional manifold and $P=M \times S U$ (2) the trivial principal $S U$ (2)-bundle. For simplicity, we assume that $\mathcal{G}$ is a Lie group that acts freely by gauge transformations on $\mathcal{A}$ and that $\mathcal{A} \rightarrow \mathcal{A} / \mathcal{G}$ is a principal $\mathcal{G}$-bundle. If $M$ is closed, then the Chern-Simons action associated with a polynomial $p \in I_{\mathbb{Z}}^{2}(S U(2))$ determines a $\mathcal{G}$-invariant function $\mathcal{A} \rightarrow \mathbb{R} / \mathbb{Z}$ and hence a function on the quotient $\mathcal{A} / \mathcal{G} \rightarrow \mathbb{R} / \mathbb{Z}$. However, when $M$ is a manifold with boundary $\partial M$, the Chern-Simons action is not a function on $\mathcal{A} / \mathcal{G}$, but it determines a section of a line bundle $\mathcal{L}_{\partial M} \rightarrow \mathcal{A} / \mathcal{G}$ called the Chern-Simons line bundle (see, e.g., [19]). Again all the constructions are based on a polynomial $p$. However, as pointed out in [12], to determine the Chern-Simons action for non-trivial bundles it is also necessary to choose a universal characteristic class $\Upsilon \in H^{4}(\mathbf{B} G)$.

We generalize these two examples to arbitrary bundles, Lie groups and dimensions in the following way. We recall (see [15]) that if $P \rightarrow M$ is a principal $G$-bundle and $\mathcal{A}$ the space of connections on $P$, the principal $G$-bundle $\mathbb{P}=P \times \mathcal{A} \rightarrow M \times \mathcal{A}$ admits a canonical (or tautological) connection $\mathbb{A}$ which is invariant under the action of the group Aut $P$ of automorphisms of $P$. If a group $\mathcal{G}$ acts on $P \rightarrow M$ by gauge transformations, then for any invariant polynomial $p \in I_{\mathbb{Z}}^{r}(G)$ we can consider the $\mathcal{G}$-equivariant characteristic forms $p_{\mathcal{G}}^{\mathbb{A}} \in \Omega_{\mathcal{G}}^{2 r}(M \times \mathcal{A})$ of $\mathbb{A}$. If $c$ is a closed oriented $d$-dimensional submanifold of $M$, by integrating $p_{\mathcal{G}}^{\mathbb{A}}$ over $c$, we obtain $\int_{c} p_{\mathcal{G}}^{\mathbb{A}} \in \Omega_{\mathcal{G}}^{2 r-d}(\mathcal{A})$ which is closed for the Cartan differential $D$. When $d=2 r-2, \varpi_{c}=\int_{c} p_{\mathcal{G}}^{\mathbb{A}} \in \Omega_{\mathcal{G}}^{2}(\mathcal{A})$ is a closed equivariant 2-form, i.e., $\varpi_{c}=\sigma_{c}+\mu_{c}$ where $\sigma_{c}$ is a closed $\mathcal{G}$-invariant 2-form and $\mu_{c}$ a co-moment map for $\sigma_{c}$. Our main result is the following

Theorem 1 Let $c$ be a closed submanifold of dimension $2 r-2$ of $M, p \in I_{\mathbb{Z}}^{r}(G), \Upsilon \in$ $H^{2 r}(\mathbf{B} G, \mathbb{Z})$ a characteristic class compatible with $p$ (i.e., they determine the same real characteristic class) and $A_{0}$ a background connection on $P$. These data determine a lift of the action of $\mathcal{G}$ on $\mathcal{A}$ to an action on $\mathcal{U}_{c}=\mathcal{A} \times U(1) \rightarrow \mathcal{A}$ by $U(1)$-bundle automorphisms, and a $\mathcal{G}$-invariant connection form $\Xi_{c}$ such that the $\mathcal{G}$-equivariant curvature of $\Xi_{c}$ is $\varpi_{c}$.

Due to the equivalence between principal $U(1)$-bundles and Hermitian line bundles, we also obtain a $\mathcal{G}$-equivariant Hermitian line bundle $\mathcal{L}_{c} \rightarrow \mathcal{A}$ with connection $\nabla^{\Xi_{c}}$. Our result also generalizes the Chern-Simons line as we prove the following result.

Proposition 2 If $c=\partial u$ for some $u \subset M$, then $S_{u}(A)=\exp \left(-2 \pi i \cdot \int_{u} T p\left(A, A_{0}\right)\right)$ determines a $\mathcal{G}$-invariant section of $\mathcal{U}_{c} \rightarrow \mathcal{A}$, or what it is equivalent, a $\mathcal{G}$-invariant section of unit norm of $\mathcal{L}_{c} \rightarrow \mathcal{A}$.

Thus $p, \Upsilon, c$ and $A_{0}$ determine a $\mathcal{G}$-equivariant prequantization bundle for $\left(\mathcal{A}, \varpi_{c}\right)$. If we change the background connection $A_{0}$, we obtain a different connection and a different 
action, but we prove that there exists a canonical $\mathcal{G}$-equivariant isomorphisms between them. Therefore, we can consider that different background connections $A_{0}$ determine different global trivializations of the same prequantization bundle, and hence that it only depends on $p, \Upsilon$ and $c$.

We prove that for any $X \in \operatorname{Lie} \mathcal{G}$, its lift $X_{\mathcal{U}_{c}} \in \mathfrak{X}\left(\mathcal{U}_{c}\right)$ does not depend on $\Upsilon$. Hence, neither does the action of the connected component of the identity $\mathcal{G}_{0}$. Nevertheless, the action of the elements of $\mathcal{G}$ which are not connected with the identity depends on $\Upsilon$. For certain groups, there is a bijection $I_{\mathbb{Z}}^{r}(G) \simeq H^{2 r}(\mathbf{B} G, \mathbb{Z})$, and in these cases the action is determined only by $c, p$ and $A_{0}$. This happens, for example, in the case $G=U(n)$ as $H^{\bullet}(\mathbf{B} U(n), \mathbb{Z}) \simeq \mathbb{Z}\left[c_{1}, \ldots, c_{n}\right]$ where $c_{1}, \ldots, c_{n}$ are the Chern classes (e.g., see [23, Chapter 23.7]). But for a general group $G$, the cohomology $H^{2 r}(\mathbf{B} G, \mathbb{Z})$ may contain torsion elements, and $\Upsilon$ is not determined by $p$. In that cases, non-equivalent actions can exist with the same $c, p$ and $A_{0}$ if $\mathcal{G}$ is not connected.

We study the dependence on $c$. It can be better understood in terms of the Hermitian line bundle $\mathcal{L}_{c} \rightarrow \mathcal{A}$. If $-c$ denotes the submanifold $c$ with the opposed orientation, then we have $\mathcal{L}_{-c}=\mathcal{L}_{c}^{*}$, and if $c^{\prime}$ is another closed oriented submanifold, then $\mathcal{L}_{c+c^{\prime}} \simeq \mathcal{L}_{c} \otimes \mathcal{L}_{c^{\prime}}$. In particular, if $\partial u=c-c^{\prime}$ by Proposition $2, S_{u}$ determines a section of unitary norm on $\mathcal{L}_{c-c^{\prime}}=\mathcal{L}_{c} \otimes \mathcal{L}_{c^{\prime}}^{*} \simeq \operatorname{Hom}\left(\mathcal{L}_{c^{\prime}}, \mathcal{L}_{c}\right)$ which is an isomorphism.

In Sect. 7.2, the restriction of the prequantization bundle $\mathcal{U}_{c}$ to the space of irreducible connections $\tilde{\mathcal{U}}_{c}=\widetilde{\mathcal{A}} \times U(1)$ is studied. We have a well-defined quotient manifold $\widetilde{\mathcal{A}} / \mathcal{G}$, and for the trivial $S U$ (2)-bundle over a Riemann surface, we have also a well-defined quotient $U(1)$-bundle $\widetilde{\mathcal{U}}_{c} / \mathcal{G} \rightarrow \widetilde{\mathcal{A}} / \mathcal{G}$. If $p$ is the second Chern polynomial and $c=M$, then $\sigma_{c}$ and $\mu_{c}$ coincide with the Atiyah-Bott symplectic structure and moment map (see [15]). The connection $\Xi_{c}$ does not project onto a connection on $\tilde{\mathcal{U}}_{c} / \mathcal{G}$ as $\iota_{X}{ }_{\mathcal{U}_{c}} \Xi_{c}=-\mu_{c}(X)$ for $X \in \mathrm{Lie} \mathcal{G}$. However, if $\widetilde{\mathcal{F}}$ is the space of irreducible flat connections, we have $\widetilde{\mathcal{F}} \subset \mu_{c}^{-1}(0)$, and the restriction of $\Xi_{c}$ to $\widetilde{\mathcal{F}} \times U(1)$ is $\mathcal{G}$-basic and projects onto a connection $\underline{\Xi}_{c}$ on $(\widetilde{\mathcal{F}} \times U(1)) / \mathcal{G} \rightarrow \widetilde{\mathcal{F}} / \mathcal{G}$. Furthermore, the curvature of $\underline{\Xi}_{c}$ is the form $\underline{\sigma}_{c}$ obtained by symplectic reduction of $\left(\mathcal{A}, \sigma_{c}, \mu_{c}\right)$. Hence, our result generalizes that of [24]. Furthermore, we also show that for other groups and bundles, the prequantization bundle of $\widetilde{\mathcal{A}} / \mathcal{G}$ is not determined by the characteristic classes of $G$, but by those of the group $\widetilde{G}=G / Z(G)$, where $Z(G)$ is the center of $G$.

The symmetry group usually considered in physical theories is the group of gauge transformations. However sometimes it is necessary to consider the lift of the action of the automorphism group Aut $P$ to $\mathcal{U}_{c}$ (see, for example, [1,2] and references therein). We show that Theorem 1 is also valid when $\mathcal{G}$ acts on $P$ by automorphisms preserving the orientation of $M$ in the following cases:

- $M$ is a closed oriented manifold of dimension $d=2 r-2$ and $c=M$.

- $M$ is a compact oriented manifold of dimension $d=2 r-1$ with boundary $\partial M$ and $c=\partial M$. In this case, Proposition 2 is also valid.

Finally, we apply our results to the space $\operatorname{Met} M$ of Riemannian metrics and the action of the orientation-preserving diffeomorphisms $\operatorname{Diff}^{+} M$. For closed manifolds of dimension $4 r-2$, the integer combinations of Pontryagin classes of degree $r$ determine Diff ${ }^{+} M-$ equivariant prequantization bundles of the presymplectic structures defined in [18]. In particular, for a surface, the first Pontryagin class is shown to determine a canonical holomorphic prequantization bundle for the Teichmüller space endowed with the Weil-Petersson symplectic form. This bundle is shown to be equivariant with respect of the action of the mapping class group of the surface. Furthermore, for compact manifolds of dimension $4 r-1$ with boundary, we obtain Chern-Simons line bundles for Riemannian metrics. 
Let us explain the way in which Theorem 1 is obtained. For simplicity, we assume that $\mathcal{G}$ is the group of gauge transformations that fixes a point $p_{0} \in P$. As it is well known, $\mathcal{G}$ acts freely on $\mathcal{A}$, and we have well-defined quotient manifolds. In [4], Chern-Weil theory is applied to the principal $G$-bundle $(P \times \mathcal{A}) / \mathcal{G} \rightarrow M \times \mathcal{A} / \mathcal{G}$. Moreover, if $p \in I_{\mathbb{Z}}^{r}(G)$, the ChernSimons construction can also be applied to this bundle. This is done in [10] by using the Cheeger-Simons approach of [11] based on differential characters. The space of differential characters of order $k$ on $M$ is denoted by $\hat{H}^{k}(M)$. If $\mathfrak{A}$ is a connection on the principal $\mathcal{G}$-bundle $\mathcal{A} \rightarrow \mathcal{A} / \mathcal{G}$, it determines a connection $\underline{\mathfrak{A}}$ on $(P \times \mathcal{A}) / \mathcal{G} \rightarrow M \times \mathcal{A} / \mathcal{G}$ (see [10] for details). Hence, if $\Upsilon \in H^{2 r}(\mathbf{B} G)$ is a universal characteristic class compatible with $p$, there exists a differential character (the Chern-Simons differential character) $\chi_{\mathfrak{A}} \in \hat{H}^{2 r}(M \times \mathcal{A} / \mathcal{G})$ whose curvature is $p\left(F_{\mathfrak{A}}\right)$. By integration over a submanifold $c$, a differential character $\int_{c} \chi_{\mathfrak{A}} \in \hat{H}^{2 r-d}(\mathcal{A} / \mathcal{G})$ is obtained, where $d=\operatorname{dim} C$. In [10], by applying our results of Sect. 3.1 , the characters of order 2 are interpreted geometrically as the holonomy of a connection on a $U(1)$-bundle $\underline{\mathcal{U}}_{c} \rightarrow \mathcal{A} / \mathcal{G}$. We generalize this construction to non-free actions, to the action of automorphisms and also to the space of Riemannian metrics.

It is possible to extend the construction of [10] to non-free actions by using equivariant cohomology. We do not follow this approach because it requires the use of connections and quotients on principal $\mathcal{G}$-bundles for infinite-dimensional groups. This can be technically difficult, especially if we want to apply it to groups of automorphisms and diffeomorphisms that should be considered as Fréchet Lie groups. To avoid this problem, we give a direct definition of the lift of the action of each element $\phi \in \mathcal{G}$. We show that it can be done using only the action of discrete groups and it does not require the use of quotients and auxiliary connections for infinite-dimensional groups. We also prove that the bundle constructed in [10] coincides with our bundle.

\section{Notations and conventions}

In this paper, we consider two Lie groups. The group $G$ is the structure group of a principal bundle $P \rightarrow M$ and it is supposed to be finite dimensional and with a finite number of connected components (in order to apply the Chern-Simons construction). The second group $\mathcal{G}$ is a symmetry group (usually infinite dimensional), and $\mathcal{G}_{0}$ denotes the connected component of the identity on $\mathcal{G}$.

We denote by $I_{\mathbb{Z}}^{r}(G)$ the set of $G$-invariant polynomials on its Lie algebra $\mathfrak{g}$ whose characteristic classes have integral periods. We denote by $\mathbf{E} G \rightarrow \mathbf{B} G$ a universal principal $G$-bundle. A polynomial $p \in I_{\mathbb{Z}}^{r}(G)$ and a characteristic class $\Upsilon \in H^{2 r}(\mathbf{B} G, \mathbb{Z})$ are said to be compatible if they determine the same real characteristic class. We denote by $\mathcal{I}_{\mathbb{Z}}^{r}=\left\{(p, \Upsilon) \in I_{\mathbb{Z}}^{r}(G) \times H^{2 r}(\mathbf{B} G, \mathbb{Z}): p, \Upsilon\right.$ are compatible $\}$, and by $\Upsilon_{P}$ the characteristic class of $P \rightarrow M$ associated with $\Upsilon$.

The Maurer-Cartan form of $U(1)$ is denoted by $\theta=u^{-1} d u$, and $\partial_{\theta} \in \mathfrak{X}(U(1))$ is the vector field such that $\theta\left(\partial_{\theta}\right)=i$. If $\pi: \mathcal{U} \rightarrow N$ is a principal $U(1)$ bundle and $\Xi \in \Omega^{1}(\mathcal{U}, i \mathbb{R})$ is a connection, then the curvature form $\operatorname{curv}(\Xi) \in \Omega^{2}(N)$ is defined by the property $\pi^{*}(\operatorname{curv}(\Xi))=\frac{i}{2 \pi} d \Xi$. The log-holonomy $\log \operatorname{hol} \Xi(\gamma) \in \mathbb{R} / \mathbb{Z}$ of $\Xi$ on a closed curve $\gamma: I \rightarrow N$ with $\gamma(0)=\gamma(1)$ is determined by the relation $\bar{\gamma}(1)=\bar{\gamma}(0) \cdot \exp \left(2 \pi i \log \operatorname{hol}_{\Xi}(\gamma)\right)$, where $\bar{\gamma}: I \rightarrow \mathcal{U}$ is a $\Xi$-horizontal lift of $\gamma$. The (real) first Chern class of $\mathcal{U}$ is the cohomology class of $\operatorname{curv}(\Xi)$. We denote by $I$ the interval $[0,1]$. 


\section{Cheeger-Simons differential characters}

We recall the definition of differential characters (see [5,11] for details). We denote by $C_{k}(N)$ and $Z_{k}(N)$ the smooth chains and cycles on $N$. A Cheeger-Simons differential character of order $k$ is a homomorphism $\chi: Z_{k-1}(N) \rightarrow \mathbb{R} / \mathbb{Z}$ such that there exist $\alpha \in \Omega^{k}(N)$ which satisfies $\chi(\partial u)=\int_{u} \alpha$ for every $u \in C_{k}(N)$. We say that $\chi$ is a differential character with curvature $\operatorname{curv}(\chi)=\alpha$, and it can be proved that $d \operatorname{curv}(\chi)=0$. We recall (e.g., see [5]) that $\chi(u)$ is invariant under reparametrizations, i.e., if $U$ is a manifold of dimension $k$ and $\varphi$ is an orientation-preserving diffeomorphism of $U$, then for any $u: U \rightarrow N$ we have $\chi(u \circ \varphi)=\chi(u)$. We denote the space of differential characters of order $k$ on $N$ by $\hat{H}^{k}(N)$. We have a map char: $\hat{H}^{k}(N) \rightarrow H^{k}(N, \mathbb{Z})$, and the class $\operatorname{char}(\chi)$ is called the characteristic class of $\chi$. The maps char and curv are compatible in the sense that we have $r(\operatorname{char}(\chi))=[\operatorname{curv}(\chi)] \in H^{k}(N, \mathbb{R})$, where $r: H^{k}(N, \mathbb{Z}) \rightarrow H^{k}(N, \mathbb{R})$ is the natural map. If $f: N^{\prime} \rightarrow N$ is a smooth map, it induces a map $f^{*}: \hat{H}^{k}(N) \rightarrow \hat{H}^{k}\left(N^{\prime}\right)$ defined by $f^{*} \chi(u)=\chi(f \circ u)$. Given $\beta \in \Omega^{k-1}(N)$ we define a differential character $\varsigma(\beta) \in \hat{H}^{k}(N)$ by setting $\varsigma(\beta)(s)=\int_{s} \beta$ for $s \in Z_{k-1}(M)$. We have $\operatorname{curv}(\varsigma(\beta))=d \beta$, and $\operatorname{char}(\varsigma(\beta))=0$. Note that $\varsigma(d \alpha)(s)=\int_{s} d \alpha=\int_{\partial s} \alpha=0$ as $\partial s=0$.

\subsection{Differential characters of order 2}

First we recall that if $\mathcal{U} \rightarrow M$ a principal $U(1)$-bundle with connection $\Theta$, and curvature $\omega \in \Omega^{2}(M)$, the log-holonomy of $\Theta \log \operatorname{hol}_{\Theta}: Z_{1}(M) \rightarrow \mathbb{R} / \mathbb{Z}$ is a differential character with curvature $\operatorname{curv}\left(\log \operatorname{hol}_{\Theta}\right)=\omega$ and $\operatorname{char}\left(\log \operatorname{hol}_{\Theta}\right)=c_{1}(\mathcal{U})$. Conversely, by a classical result in differential cohomology, every second-order differential character can be represented as the holonomy of a connection $\Theta$ on a principal $U(1)$ bundle $\mathcal{U} \rightarrow M$. The bundle $\mathcal{U}$ and the connection $\Theta$ are determined by $\chi$ only modulo isomorphisms. In the next Proposition, we show that in the following restrictive equivariant case it is possible to give a concrete bundle and connection

Theorem 3 Let $N$ be a connected manifold with $H_{1}(N, \mathbb{Z})=0$ in which $\mathcal{G}$ acts in such a way that $\pi: N \rightarrow N / \mathcal{G}$ is a principal $\mathcal{G}$-bundle. Let $\chi \in \hat{H}^{2}(N / \mathcal{G})$ be a second-order differential character on $N / \mathcal{G}$ with curvature $\omega$ and assume that there exists $\lambda \in \Omega^{1}(N)$ such that $\pi^{*} \omega=d \lambda$. Then, there exists a unique lift of the action of $\mathcal{G}$ to $N \times U(1)$ by $U(1)$-bundle automorphism such that $\Theta=\theta-2 \pi i \lambda \in \Omega^{1}(N \times U(1), i \mathbb{R})$ is projectable onto a connection $\underline{\Theta}$ on $\mathcal{U}=(N \times U(1)) / \mathcal{G} \rightarrow N / \mathcal{G}$ and $\chi=\log \operatorname{hol}_{\underline{\Theta}}$. The action of $\phi \in \mathcal{G}$ on $N \times U(1)$ is given by $\Phi_{\phi}: N \times U(1) \rightarrow N \times U(1), \Phi_{\phi}(x, u)=\left(\phi \bar{x}, \exp \left(2 \pi i \alpha_{\phi}(x)\right) \cdot u\right)$, where $\alpha_{\phi}: N \rightarrow \mathbb{R} / \mathbb{Z}$ is defined by $\alpha_{\phi}(x)=\int_{\gamma} \lambda-\chi(\pi \circ \gamma)$, and $\gamma$ is any curve on $N$ joining $x$ and $\phi x$.

Theorem 3 is a consequence of the following lemmas. If $\gamma$ is a curve on $N$, we denote by $\underline{\gamma}=\pi \circ \gamma$ the projected curve on $N / \mathcal{G}$.

Lemma 4 Let $\gamma$ and $\gamma^{\prime}$ be two curves on $N$ joining $x$ and $\phi x$. Then $\int_{\gamma} \lambda-\chi(\underline{\gamma})=\int_{\gamma^{\prime}} \lambda-$ $\chi\left(\underline{\gamma^{\prime}}\right)$.

Proof As $H_{1}(N, \mathbb{Z})=0$, we have $\gamma-\gamma^{\prime}=\partial D$ on $N$. If $\underline{D}=\pi \circ D$, then $\underline{\gamma}-\gamma^{\prime}=\partial \underline{D}$, and hence $\chi(\underline{\gamma})-\chi\left(\underline{\gamma}^{\prime}\right)=\chi\left(\underline{\gamma}-\underline{\gamma}^{\prime}\right)=\int_{\underline{D}} \omega=\int_{D} d \lambda=\int_{\gamma} \lambda-\int_{\gamma^{\prime}} \lambda$.

For every $\phi \in \mathcal{G}$, we define $\alpha_{\phi}: N \rightarrow \mathbb{R} / \mathbb{Z}$ by $\alpha_{\phi}(x)=\int_{\gamma} \lambda-\chi(\gamma)$, where $\gamma$ is a curve on $N$ joining $x$ and $\phi x$. (It is well defined by the preceding Lemma.) 
Lemma 5 (a) We have $\alpha_{\phi}\left(x^{\prime}\right)=\alpha_{\phi}(x)+\int_{\gamma_{x x^{\prime}}}\left(\phi^{*} \lambda-\lambda\right)$ for any curve $\gamma_{x x^{\prime}}$ joining $x$ and $x^{\prime}$.

(b) As a consequence of a), we have $d \alpha_{\phi}=\phi^{*} \lambda-\lambda$.

(c) If $\phi_{t}$ is a 1-parameter group on $\mathcal{G}$ with $\dot{\phi}=X$, then $\left.\frac{d \alpha_{\phi_{t}}(x)}{d t}\right|_{t=0}=\lambda\left(X_{N}\right)(x)$.

Proof (a) If $\gamma$ is a curve joining $x$ and $\phi x, \gamma_{x^{\prime} x}$ a curve joining $x^{\prime}$ and $x$, and $\gamma_{x x^{\prime}}$ the inverse curve. Then $\gamma^{\prime}=\gamma_{x^{\prime} x} * \gamma * \phi \gamma_{x x^{\prime}}$ is a curve joining $x^{\prime}$ and $\phi x^{\prime}$. Clearly, $\underline{\gamma}^{\prime}=\gamma$ in $Z_{1}(N / \mathcal{G})$, and hence $\chi(\underline{\gamma})=\chi\left(\underline{\gamma}^{\prime}\right)$. We have

$$
\begin{aligned}
\alpha_{\phi}\left(x^{\prime}\right) & =\int_{\gamma^{\prime}} \lambda-\chi\left(\underline{\gamma^{\prime}}\right)=\int_{\gamma_{x^{\prime} x}} \lambda+\int_{\gamma} \lambda+\int_{\phi \gamma_{x x^{\prime}}} \lambda-\chi \underline{(\underline{\gamma})} \\
& =\alpha_{\phi}(x)+\int_{\gamma_{x x^{\prime}}} \phi^{*} \lambda-\int_{\gamma_{x x^{\prime}}} \lambda=\alpha_{\phi}(x)+\int_{\gamma_{x x^{\prime}}}\left(\phi^{*} \lambda-\lambda\right)
\end{aligned}
$$

(b) If $\gamma$ is a curve with $\gamma(0)=x$ and $\gamma^{\prime}(0)=X \in T_{x} N$, we have $\alpha_{\phi}(\gamma(s))=\alpha_{\phi}(x)+$ $\int_{0}^{s}\left(\phi^{*} \lambda-\lambda\right)_{\gamma(s)}\left(\gamma^{\prime}(s)\right) d s$, and hence $d \alpha_{\phi}(x)(X)=\left.\frac{d \alpha_{\phi}\left(x_{s}\right)}{d s}\right|_{s=0}=\left(\phi^{*} \lambda-\lambda\right)_{x}(X)$.

(c) Let $X \in \operatorname{Lie} \mathcal{G}$ and $\phi_{t}$ a 1-parameter group on $\mathcal{G}$ with $\phi_{0}=1_{\mathcal{G}}$ and $\dot{\phi}_{0}=X$, and we define $\gamma(t)=\phi_{t} x$. We have $\underline{\gamma}=0$ in $Z_{1}(N / \mathcal{G})$ and $\dot{\gamma}(0)=X_{N}(x)$. Then $\alpha_{\phi_{t}}(x)=$ $\left.\int_{\gamma} \lambda-\chi \underline{\gamma}\right)=\int_{\gamma} \lambda=\int_{0}^{t} \lambda_{\phi_{s} x}(\dot{\gamma}(s)) d s$ and $\left.\frac{\mathrm{d} \alpha_{\phi_{t}}(x)}{\mathrm{d} t}\right|_{t=0}=\lambda\left(X_{N}(x)\right)$.

The action $\alpha$ satisfies the following cocycle condition

Lemma 6 We have $\alpha_{\phi_{2} \phi_{1}}(x)=\alpha_{\phi_{1}}(x)+\alpha_{\phi_{2}}\left(\phi_{1} x\right)$ for any $x \in N, \phi_{1}, \phi_{2} \in \mathcal{G}$.

Proof Let $\gamma_{1}$ be a curve joining $x$ and $\phi_{1} x$ and $\gamma_{2}$ be a curve joining $\phi_{1} x$ and $\phi_{2} \phi_{1} x$. Then $\gamma^{\prime}=\gamma_{2} * \gamma_{1}$ is a curve joining $x$ and $\phi_{2} \phi_{1} x$. We have $\underline{\gamma}^{\prime}=\underline{\gamma}_{2}+\underline{\gamma}_{1}$ in $Z_{1}(N / \mathcal{G})$. Hence

$$
\alpha_{\phi_{2} \phi_{1}}(x)=\int_{\gamma^{\prime}} \lambda-\chi\left(\underline{\gamma}^{\prime}\right)=\int_{\gamma_{2}} \lambda+\int_{\gamma_{1}} \lambda-\chi\left(\underline{\gamma}_{1}\right)-\chi\left(\underline{\gamma}_{2}\right)=\alpha_{\phi_{1}}(x)+\alpha_{\phi_{2}}\left(\phi_{1} x\right) \text {. }
$$

We define the action of $\mathcal{G}$ on $N \times U(1)$ by $\Phi_{\phi}(x, u)=\left(\phi x, \exp \left(2 \pi i \alpha_{\phi}(x)\right) \cdot u\right)$. It defines a group action as we have

$$
\begin{aligned}
\Phi_{\phi_{2}}\left(\Phi_{\phi_{1}}(x, u)\right) & =\Phi_{\phi_{2}}\left(\left(\phi_{1} x, \exp \left(2 \pi i \alpha_{\phi_{1}}(x)\right) \cdot u\right)\right) \\
& =\left(\phi_{2} \phi_{1} x, \exp \left(2 \pi i\left(\alpha_{\phi_{1}}(x)+\alpha_{\phi_{2}}\left(\phi_{1} x\right)\right) \cdot u\right)\right. \\
& \left.=\left(\phi_{2} \phi_{1} x, \exp \left(2 \pi i \alpha_{\phi_{2} \phi_{1}}(x)\right) \cdot u\right)=\left(\Phi_{\phi_{2} \phi_{1}}\right)(x, u)\right)
\end{aligned}
$$

We also define $\Theta=\theta-2 \pi i \lambda \in \Omega^{1}(N \times U(1), i \mathbb{R}), \mathcal{U}=(N \times U(1)) / \mathcal{G}$, and we denote by $\bar{\pi}: N \times U(1) \rightarrow \mathcal{U}$ the projection. For every $\phi \in \mathcal{G}$, we have

$$
\Phi^{*} \Theta=\Phi^{*} \theta-2 \pi i \phi^{*} \lambda=\left(\theta+2 \pi i d \alpha_{\phi}\right)-2 \pi i \phi^{*} \lambda=\left(\theta+2 \pi i\left(\phi^{*} \lambda-\lambda\right)\right)-2 \pi i \phi^{*} \lambda=\Theta .
$$

Moreover, for every $X \in \operatorname{Lie} \mathcal{G}$, if $\phi_{t}$ is a curve on $\mathcal{G}$ with $X=\dot{\phi}$, then we have $X_{N \times U(1)}=$ $X_{N}+\left.2 \pi i \frac{\mathrm{d} \alpha_{\phi_{t}}(x)}{\mathrm{d} t}\right|_{t=0} \partial_{\theta}=X_{N}+2 \pi i \lambda\left(X_{N}\right) \partial_{\theta}$, and hence, $\Theta\left(X_{N \times U(1)}\right)=0$. We conclude that $\Theta$ is a $\mathcal{G}$-basic form, i.e., there exists $\underline{\Theta} \in \Omega^{1}(N / \mathcal{G}, i \mathbb{R})$ such that $\bar{\pi}^{*} \underline{\Theta}=\Theta$. Clearly, $\Theta$ is a connection form.

Lemma 7 We have $\log \operatorname{hol}_{\underline{\Theta}}=\chi$. 
Proof Given a loop $\underline{\gamma}$ on $N / \mathcal{G}$ with $\underline{\gamma}(0)=\underline{\gamma}(1)=[x] \in N / \mathcal{G}$, we can find a curve $\gamma$ on $N$ with $\gamma(0)=x$ such that $\pi \circ \gamma=\underline{\gamma}$. We have $\gamma(1)=\phi x$ for some $\phi \in \mathcal{G}$. The $\Theta$-horizontal lift of $\gamma$ to $N \times U(1)$ starting at the point $(x, 0)$ is given by $\bar{\gamma}(s)=$ $\left(\gamma(s), \exp \left(2 \pi i \int_{0}^{s} \lambda_{\gamma(t)}(\dot{\gamma}(t)) \mathrm{d} t\right)\right)$. The curve $\bar{\pi} \circ \bar{\gamma}$ is a $\underline{\Theta}$-horizontal lift to $\mathcal{U}$ of the loop $\underline{\gamma}=$ $\pi \circ \gamma$. In particular, we have $\bar{\pi} \circ \bar{\gamma}(1)=\left(\phi x, \exp \left(2 \pi i \int_{\gamma} \lambda\right)\right) \sim_{\mathcal{G}}\left(x, \exp \left(2 \pi i\left(\int_{\gamma} \lambda-\alpha_{\phi}(\bar{x})\right)\right)\right.$. Hence $\log \operatorname{hol}_{\underline{\Theta}}(\underline{\gamma})=\int_{\gamma} \lambda-\alpha_{\phi}(x)=\chi(\underline{\gamma})$.

The proof of the preceding Proposition also shows that the action we have defined is the unique which satisfies $\log \operatorname{hol}_{\underline{\Theta}}(\underline{\gamma})=\chi(\underline{\gamma})$. This is equivalent to $\chi(\underline{\gamma})=\int_{\gamma} \lambda-\alpha_{\phi}(x)$, and hence $\alpha_{\phi}(x)=\int_{\gamma} \lambda-\chi(\gamma)$, that is our definition of $\alpha_{\phi}(x)$.

For the elements in $\mathcal{G}_{0}$ (the connected component of the identity in $\left.\mathcal{G}\right)$, we have a simpler result:

Proposition 8 Let $\phi \in \mathcal{G}_{0}$ and $\varphi \subset \mathcal{G}$ be a curve such that $\varphi_{0}=1_{\mathcal{G}}$ and $\varphi_{1}=\phi$. Then $\alpha_{\phi}(x)=\int_{\varphi \cdot x} \lambda$.

Proof The curve $\gamma=\varphi \cdot x$ is a curve joining $x$ and $\phi x$, and $\underline{\gamma}=0$ in $Z_{1}(N / \mathcal{G})$. Hence $\alpha_{\phi}(x)=\int_{\gamma} \lambda-\chi(\underline{\gamma})=\int_{\varphi x} \lambda$.

Remark 9 The preceding Proposition determines the action of $\mathcal{G}_{0}$ only in terms of $\lambda$, and without any reference to $\chi$. Hence the differential character $\chi$ is necessary only to determine the action of the elements of $\mathcal{G}$ not connected to the identity. We note that Theorem 3 is a generalization to non-connected groups of results in $[8,14,25]$ for the space of connections.

\subsection{Chern-Simons differential characters}

Chern-Simons theory allows us to construct, in a natural way, a differential character with curvature a characteristic form. Let $G$ be a Lie group with a finite number of connected components, $p \in I_{\mathbb{Z}}^{r}(G)$ and $q: P \rightarrow N$ a principal $G$-bundle over a manifold $N$. If $A$ is a connection on $q: P \rightarrow N$ with curvature $F$, we have $p(F) \in \Omega^{k}(N)$. It can be seen (see [11]) that if $\Upsilon \in H^{2 r}(\mathbf{B} G, \mathbb{Z})$ is a universal characteristic class compatible with $p$, there exists a differential character $\chi_{A} \in \hat{H}^{2 r}(N)$ such that $\operatorname{curv}\left(\chi_{A}\right)=p(F)$ and $\operatorname{char}\left(\chi_{A}\right)=\Upsilon_{P}$. We call $\chi_{A}$ the Chern-Simons character of $p, \Upsilon$ and $A$. The Chern-Simons character is characterized as being the unique natural map $(P, A) \mapsto \chi_{A}$ satisfying $\operatorname{curv}\left(\chi_{A}\right)=p(F)$ and $\operatorname{char}\left(\chi_{A}\right)=\Upsilon_{P}$. We recall that natural means that for any principal $G$-bundle $P^{\prime} \rightarrow N^{\prime}$ and any $G$-bundle map $F: P^{\prime} \rightarrow P$ we have $\chi_{F^{*} A}=f^{*}\left(\chi_{A}\right)$, where $f: N^{\prime} \rightarrow N$ is the map induced by $F$. Furthermore, if $A^{\prime}$ is another connection on $P$, then we have

$$
\chi_{A^{\prime}}=\chi_{A}+\varsigma\left(\operatorname{Tp}\left(A^{\prime}, A\right)\right) .
$$

A consequence of the preceding equation is the following (see, e.g., [11, Proposition 2.9])

Lemma 10 If $A_{t}$ is a smooth 1-parametric family of connections on $P$ with $\dot{A}_{0}=a \in$ $\Omega^{1}(M, \operatorname{ad} P)$, then $\left.\frac{d}{d t}\right|_{t=0} \chi_{A_{t}}(u)=r \int_{u} p\left(a, F_{0},{ }^{(r-1)}, F_{0}\right)$ for every $u \in Z_{2 r-1}(N)$.

Remark 11 The original Chern-Simons and Cheeger-Simons constructions are valid for finite-dimensional manifolds, but they can be extended to Banach or Fréchet infinitedimensional manifolds, and to more general types of spaces (see, for example, [5]). Hence, they can be applied to the infinite-dimensional spaces of connections and metrics. 


\subsection{Fiber integration of differential characters}

\subsubsection{Integration on a product}

If $\alpha \in \Omega^{k}(C \times S)$ with $C$ compact and $\operatorname{dim} C=d$, we define $\int_{C} \alpha \in \Omega^{k-d}(S)$ by $\left(\int_{C} \alpha\right)_{s}\left(X_{1}, \ldots, X_{k-d}\right)=\int_{C} \iota_{X_{k-d}} \cdots \iota_{X_{1}} \alpha_{s}$ for $s \in S, X_{1}, \ldots, X_{d} \in T_{s} S$. If $k<d$ we define $\int_{C} \alpha=0$. We have $\int_{S} \int_{C} \alpha=\int_{C \times S} \alpha$, and also $\int_{C} \alpha=\int_{C} \alpha^{d, k-d}$, where $\alpha^{d, k-d}$ is the component relative to the bigrading associated with the product structure on $C \times S$. Furthermore, we have Stokes theorem $d \int_{C} \alpha=\int_{C} d \alpha-(-1)^{k-d} \int_{\partial C} \alpha$. If $c: C \rightarrow M$ is a map with $\operatorname{dim} C=d$, we define maps $\int_{c}: \Omega^{k}(M \times N) \rightarrow \Omega^{k-d}(N)$ by $\int_{c} \alpha=\int_{C}\left(c \times \operatorname{id}_{N}\right)^{*} \alpha$ and we have $\int_{c} \alpha=\int_{c} \alpha^{d, k-d}$.

The integration map can be extended to differential characters in the following way. If $\chi \in \hat{H}^{n}(M \times N)$ is a differential character of order $n$ on $M \times N$ and $c: C \rightarrow M$ is a smooth map with $C$ closed, we define $\int_{c} \chi \in \hat{H}^{n-d}(N)$ by $\left(\int_{c} \chi\right)(s)=\chi(c \times s)$, and we have $\int_{c} \chi(\partial t)=\chi(c \times \partial t)=\int_{c \times t} \operatorname{curv}(\chi)=\int_{t} \int_{c} \operatorname{curv}(\chi)$. Hence, $\int_{c} \chi$ is a differential character on $N$ and its curvature is $\operatorname{curv}\left(\int_{c} \chi\right)=\int_{c} \operatorname{curv}(\chi)$. Moreover, if $c=\partial u$ for some $u: U \rightarrow M$, we have $\int_{\partial u} \chi=\varsigma\left(\int_{u} \operatorname{curv}(\chi)\right)$ as $\int_{\partial u} \chi(s)=\chi(\partial u \times s)=\chi(\partial(u \times s))=$ $\int_{u \times s} \operatorname{curv}(\chi)=\int_{s} \int_{u} \operatorname{curv}(\chi)$.

\subsubsection{Fiber integration}

The integration of differential characters can be extended to fiber integration on a non-trivial bundle $\mathcal{N} \rightarrow N$ with fiber $M$ (e.g., see [5,20]). In the product case, we can integrate over any submanifold of $M$, but for non-trivial bundles it only makes sense to integrate over the fiber $M$ and over $\partial M$ if the fiber has boundary. If $\mathcal{N} \rightarrow N$ is a fiber bundle with compact and oriented fiber $M$ without boundary of dimension $d$, the fiber integration is a map $\int_{M}: \hat{H}^{n}(\mathcal{N}) \rightarrow$ $\hat{H}^{n-d}(N)$ and satisfies $\operatorname{curv}\left(\int_{M} \chi\right)=\int_{M} \operatorname{curv}(\chi)$, and $\operatorname{char}\left(\int_{M} \chi\right)=\int_{M} \operatorname{char}(\chi)$. If $M$ has boundary, we have a map $\int_{\partial M}: \hat{H}^{n}(\mathcal{N}) \rightarrow \hat{H}^{n-d+1}(N)$ and

$$
\int_{\partial M} \chi=\varsigma\left(\int_{M} \operatorname{curv}(\chi)\right) \text {. }
$$

Fiber integration satisfies the following naturality property (e.g., see [5]): If $f: N^{\prime} \rightarrow N$ is a smooth map, $f^{*} \mathcal{N} \rightarrow N^{\prime}$ is the pullback bundle and $\hat{f}: f^{*} \mathcal{N} \rightarrow \mathcal{N}$ is the induced map, then we have $\int_{M} \hat{f}^{*} \chi=f^{*}\left(\int_{M} \chi\right)$. If $M$ has boundary, we have $\int_{\partial M} \hat{f}^{*} \chi=f^{*}\left(\int_{\partial M} \chi\right)$.

If $F: \mathcal{N}^{\prime} \rightarrow \mathcal{N}$ is a morphism of bundles with $f: N^{\prime} \rightarrow N$ being the induced map of the base, then we have $F=\hat{f} \circ \tilde{F}$ for a bundle morphism $\tilde{F}: \mathcal{N}^{\prime} \rightarrow f^{*} \mathcal{N}$ covering the identity map. If $\tilde{F}$ is an isomorphism of bundles that preserves the orientation on the fibers, then we have $\int_{M} \tilde{F}^{*} \chi=\int_{M} \chi$ for any $\chi \in \hat{H}^{k}\left(f^{*} \mathcal{N}\right)$. Hence, we have the following

Proposition 12 Let $\mathcal{N}^{\prime} \rightarrow N^{\prime}$ and $\mathcal{N} \rightarrow N$ be bundles with fiber $M$ and let $G: \mathcal{N}^{\prime} \rightarrow \mathcal{N}$ be a morphism covering the map $f: N^{\prime} \rightarrow N$. If $\tilde{F}: \mathcal{N}^{\prime} \rightarrow f^{*} \mathcal{N}$ is an isomorphism of bundles that preserves the orientation on the fibers, then for any $\chi \in \hat{H}^{n}(\mathcal{N})$ we have $\int_{M} F^{*} \chi=f^{*}\left(\int_{M} \chi\right)$. If $M$ has boundary, we have $\int_{\partial M} F^{*} \chi=f^{*}\left(\int_{\partial M} \chi\right)$.

\section{Equivariant deRham cohomology in the Cartan model}

We recall the definition of equivariant cohomology in the Cartan model (e.g., see [7,22]). Suppose that we have a left action of a connected Lie group $\mathcal{G}$ on a manifold $N$. The map 
$X \mapsto X_{N}(x)=\left.\frac{\mathrm{d}}{\mathrm{d} t}\right|_{t=0}(\exp (-t X))(x)$ induces a Lie algebra homomorphism Lie $\mathcal{G} \rightarrow$ $\mathfrak{X}(N)$. The space of $\mathcal{G}$-equivariant differential forms is the space of $\mathcal{G}$-invariant polynomials on Lie $\mathcal{G}$ with values in $\Omega^{\bullet}(N), \Omega_{\mathcal{G}}(N)=\left(\mathbf{S}^{\bullet}\left(\operatorname{Lie} \mathcal{G}^{*}\right) \otimes \Omega^{\bullet}(N)\right)^{\mathcal{G}}(\mathcal{G}$ acts on Lie $\mathcal{G}$ by the adjoint representation). The gradation on $\Omega_{\mathcal{G}}(N)$ is defined by setting $\operatorname{deg}(\alpha)=2 k+$ $r$ if $\alpha \in \mathbf{S}^{k}\left(\operatorname{Lie} \mathcal{G}^{*}\right) \otimes \Omega^{r}(N)$. Let $D: \Omega_{\mathcal{G}}^{q}(N) \rightarrow \Omega_{\mathcal{G}}^{q+1}(N)$ be the Cartan differential, $(D \alpha)(X)=d(\alpha(X))-\iota_{X_{N}} \alpha(X)$, for $X \in \operatorname{Lie} \mathcal{G}$. On $\Omega_{\mathcal{G}}^{\bullet}(N)$, we have $D^{2}=0$, and the $\mathcal{G}$-equivariant cohomology (in the Cartan model) of $N$ is defined as the cohomology of this complex.

A $\mathcal{G}$-equivariant 2-form $\varpi$ is given by $\varpi(X)=\sigma+\mu(X)$ where $\sigma$ is a $\mathcal{G}$-invariant 2-form and $\mu:$ Lie $\mathcal{G} \rightarrow \Omega^{0}(N)$ a linear $\mathcal{G}$-equivariant map. The form $\varpi$ is $D$-closed if $d \sigma=0$ and $\iota^{\chi_{N}} \sigma=\mu(X)$ for every $X$. Hence, $\mu$ is a co-moment map for $\sigma$.

If a group acts on $M, N, C$ and $c: C \rightarrow M$ is $\mathcal{G}$-equivariant, the integration map is extended to equivariant differential forms $\int_{c}: \Omega_{\mathcal{G}}^{k}(M \times N) \rightarrow \Omega_{\mathcal{G}}^{k-d}(N)$ by setting $\left(\int_{c} \alpha\right)(X)=$ $\int_{c}(\alpha(X))$ for $X \in \operatorname{Lie} \mathcal{G}$, and we have $D \int_{C} \alpha=\int_{C} D \alpha-(-1)^{k-d} \int_{\partial C} \alpha$.

\subsection{Equivariant characteristic classes in the Cartan model}

We recall the definition of equivariant characteristic classes (see [6,9] for details). Let $\mathcal{G}$ be a group that acts (on the left) on a principal $G$-bundle $\pi: P \rightarrow M$ and let $A$ be a connection on $P$ invariant under the action of $\mathcal{G}$. It can be proved (see $[6,9]$ ) that for every $X \in \operatorname{Lie} \mathcal{G}$ the $\mathfrak{g}$-valued function $A\left(X_{P}\right)$ is of adjoint type and defines a section of the adjoint bundle $v_{A}(X) \in \Omega^{0}(N$, ad $P)$. For every $p \in I^{r}(G)$ the $\mathcal{G}$-equivariant characteristic form $p_{\mathcal{G}}^{A} \in \Omega_{\mathcal{G}}^{2 k}(N)$ associated with $p$ and $A$, is defined by $p_{\mathcal{G}}^{A}(X)=p\left(F_{A}-v_{A}(X)\right)$ for every $X \in \mathrm{Lie} \mathcal{G}$.

A $\mathcal{G}$-equivariant $U(1)$-bundle is a principal $U(1)$-bundle $\mathcal{U} \rightarrow N$ in which $\mathcal{G}$ acts by $U$ (1)-bundle automorphisms. If $\Xi \in \Omega^{1}(\mathcal{U}, i \mathbb{R})$ is a $\mathcal{G}$-invariant connection, then $\frac{i}{2 \pi} D(\Xi)$ projects onto a closed $\mathcal{G}$-equivariant 2-form $\operatorname{curv}_{\mathcal{G}}(\Xi) \in \Omega_{\mathcal{G}}^{2}(N)$ called the $\mathcal{G}$-equivariant curvature of $\Xi$. If $X \in \operatorname{Lie} \mathcal{G}$, then $\operatorname{curv}_{\mathcal{G}}(\Xi)(X)=\operatorname{curv}(\Xi)-\frac{i}{2 \pi} \iota_{X} X_{\mathcal{U}} \Xi$. If $\varpi \in \Omega_{\mathcal{G}}^{2}(N)$, a $\mathcal{G}$-equivariant prequantization bundle for $\varpi$ is a principal $U(1)$-bundle $\mathcal{U} \rightarrow N$ with a $\mathcal{G}$-invariant connection $\Xi$ such that $\operatorname{curv}_{\mathcal{G}}(\Xi)=\varpi$.

\section{The space of connections}

Let $P \rightarrow M$ be a principal $G$-bundle, and $\mathcal{A}$ the space of principal connections on this bundle. As $\mathcal{A}$ is an affine space modeled on $\Omega^{1}(M, \operatorname{ad} P)$, we have canonical isomorphisms $T_{A} \mathcal{A} \simeq$ $\Omega^{1}(M, \operatorname{ad} P)$ for any $A \in \mathcal{A}$. The Lie algebra of Aut $P$ is the space of $G$-invariant vector fields on $P$, aut $P \subset \mathfrak{X}(P)$, and the Lie algebra of Gau $P$ is the subspace gau $P$ of vertical $G$-invariant vector fields. We have an identification gau $P \simeq \Omega^{0}(M, \operatorname{ad} P)$. The group Aut $P$ acts on $\mathcal{A}$ and for any $X \in$ aut $P$ we have $X_{\mathcal{A}}(A)=d^{A}\left(v_{A}(X)\right)$. In particular, if $X \in \operatorname{gau} P \simeq \Omega^{0}(M, \operatorname{ad} P)$ we have $v_{A}(X) \simeq X$ and $X_{\mathcal{A}}(A)=d^{A} X$. The principal $G$-bundle $\mathbb{P}=P \times \mathcal{A} \rightarrow M \times \mathcal{A}$ has a tautological connection $\mathbb{A} \in \Omega^{1}(P \times \mathcal{A}, \mathfrak{g})$ defined by $\mathbb{A}_{(x, A)}(X, Y)=A_{x}(X)$ for $(x, A) \in P \times \mathcal{A}, X \in T_{x} P, Y \in T_{A} \mathcal{A}$. We denote by $\mathbb{F}$ the curvature of $\mathbb{A}$, and we have $\mathbb{F}_{(x, A)}\left(a, a^{\prime}\right)=0, \mathbb{F}_{(x, A)}(a, Y)=a(Y), \mathbb{F}_{(x, A)}\left(Y, Y^{\prime}\right)=F_{A}\left(Y, Y^{\prime}\right)$ for $Y, Y^{\prime} \in T_{x} M$, and $a, a^{\prime} \in T_{A} \mathcal{A} \simeq \Omega^{1}(M$, ad $P)$. The group Aut $P$ acts on $\mathbb{P}$ by automorphisms and $\mathbb{A}$ is an Aut $P$-invariant connection. As the connection $\mathbb{A}$ is Aut $P$-invariant, for any $p \in I^{r}(G)$ we can define the Aut $P$-equivariant characteristic form $p_{\text {Aut } P}^{\mathbb{A}} \in \Omega_{\text {Aut } P}^{2 r}(M \times \mathcal{A})$, given by $p_{\text {Aut } P}^{\mathbb{A}}(X)=p\left(\mathbb{F}-v_{\mathbb{A}}(X)\right)$ for $X \in$ aut $P$. If $M$ is a closed oriented manifold of dimension $n$ 
and we consider the action of the group $\mathrm{Aut}^{+} P, p_{\text {Aut }^{+} P}^{\mathbb{A}} \in \Omega_{\text {Aut }^{+} P}^{2 r}(M \times \mathcal{A})$ can be integrated over $M$ to obtain $\int_{M} p_{\text {Aut }^{+} P}^{\mathbb{A}} \in \Omega_{\text {Aut }^{+} P}^{2 r-n}(\mathcal{A})$. In particular, if $n=2 r-2$, we have $\varpi_{M}=$ $\int_{M} p_{\mathrm{Aut}^{+} P}^{\mathrm{A}} \in \Omega_{\mathrm{Aut}^{+} P}^{2}(\mathcal{A})$ that can be written $\varpi_{M}=\sigma_{M}+\mu_{M}$, with $\mu_{M}$ a co-moment map for $\sigma_{M}$. If $M$ has boundary, we can integrate over $\partial M$ and we obtain $\int_{\partial M} p_{\text {Aut }^{+} P}^{\mathbb{A}} \in \Omega_{\text {Aut }^{+} P}^{2 r-n+1}(\mathcal{A})$. In particular, if $n=2 r-1$, we have $\varpi_{\partial M}=\int_{\partial M} p_{\text {Aut }^{+} P}^{\mathbb{A}} \in \Omega_{\text {Aut }^{+} P}^{2}(\mathcal{A})$ that can be written $\varpi_{\partial M}=\sigma_{\partial M}+\mu_{\partial M}$, with $\mu_{\partial M}$ a co-moment map for $\sigma_{\partial M}$.

If we consider the action of the Gauge group, we have the Gau $P$-equivariant characteristic form $p_{\mathrm{Gau} P}^{\mathbb{A}} \in \Omega_{\mathrm{Gau} P}^{2 r}(M \times \mathcal{A})$, given by $p_{\mathrm{Gau} P}^{\mathbb{A}}(X)=p(\mathbb{F}-X)$ for $X \in \operatorname{gau} P$. If $C$ is a closed and oriented manifold of dimension $d$, for any map $c: C \rightarrow M$ we can integrate $\left(c \times \operatorname{id}_{\mathcal{A}}\right)^{*} p_{\mathrm{Gau} P}^{\mathbb{A}} \in \Omega_{\mathrm{Gau} P}^{2 r}(C \times \mathcal{A})$ over $C$ to obtain $\int_{c} p_{\mathrm{Gau} P}^{\mathbb{A}} \in \Omega_{\mathrm{Gau} P}^{2 r-d}(\mathcal{A})$. Again if $d=2 r-2$, we have $\varpi_{c}=\int_{c} p_{\mathrm{Gau} P}^{\mathbb{A}} \in \Omega_{\mathrm{Gau} P}^{2}(\mathcal{A})$ that can be written $\varpi_{c}=\sigma_{c}+\mu_{c}$, with $\mu_{c}$ a co-moment map for $\sigma_{c}$.

The explicit expression of these forms is the following (see [15]). For $A \in \mathcal{A}, a, b \in$ $T_{A} \mathcal{A} \simeq \Omega^{1}(M, \operatorname{ad} P)$ and $X \in$ aut $P$, we have $\left(\sigma_{M}\right)_{A}(a, b)=$ $r(r-1) \int_{M} p\left(a, b, F_{A},{ }^{(r-2)}, F_{A}\right)$ and $\left(\mu_{M}\right)_{A}(X)=-r \int_{M} p\left(v_{A}(X), F_{A}, \stackrel{(r-1)}{*}, F_{A}\right)$, and similar expressions for $\partial M$ and $c$.

As commented in Introduction, our objective in this paper is to obtain equivariant prequantization bundles of $\left(\mathcal{A}, \varpi_{M}\right),\left(\mathcal{A}, \varpi_{\partial M}\right)$ and $\left(\mathcal{A}, \varpi_{c}\right)$.

\section{Equivariant prequantization bundle}

In this section, we define the equivariant prequantization bundle. In Sect. 6.1, we define the equivariant prequantization bundle for the action of a discrete group. We show in Sect. 6.2 that the definition for an arbitrary group can be reduced to the discrete case. In place of working with the space of connections $\mathcal{A}$, we consider a general connected and simply connected manifold $N$. It includes as particular cases the space of connections for $N=\mathcal{A}$, and also the case of the space of Riemannian metrics.

We assume that $\mathcal{G}$ is a Lie group that acts (on the left) on the following spaces

(a) on a principal $G$-bundle $P \rightarrow M$ by $G$-bundle automorphisms,

(b) on a connected and simply connected manifold $N$ and $\mathbb{A}$ is a $\mathcal{G}$-invariant connection on the product bundle $\mathbb{P}=P \times N \rightarrow M \times N$.

(c) on a closed oriented manifold $C$ of dimension $d=2 r-2$ and we have a $\mathcal{G}$-equivariant map $c: C \rightarrow M$ and $\mathcal{G}$ preserves the orientation of $C$. We are interested in the following cases

(c $\left.c_{1}\right)$ The action of $\mathcal{G}$ on $M$ and $C$ is trivial (i.e., $\mathcal{G}$ acts on $P$ by gauge transformations). In this case, we can consider any map $c: C \rightarrow M$.

$\left(c_{2}\right) \quad M$ is compact, $\partial M=\emptyset$ and $\mathcal{G}$ preserves the orientation on $M$. In this case, we can take $C=M$ and $c=\mathrm{id}_{M}$.

(c) $M$ is an oriented manifold with compact boundary $\partial M$ and $\mathcal{G}$ preserves the orientation on $M$. In this case, we take $C=\partial M$ and $c$ the inclusion $c: \partial M \hookrightarrow M$.

The following definitions are generalizations of the results in [15] for connections and [18] for Riemannian metrics.

As the connection $\mathbb{A}$ is $\mathcal{G}$-invariant, for any polynomial $p \in I^{r}(G)$ we can define the $\mathcal{G}$-equivariant characteristic class $p_{\mathcal{G}}^{\mathbb{A}} \in \Omega_{\mathcal{G}}^{2 r}(M \times N)$, given by $p_{\mathcal{G}}^{\mathbb{A}}(X)=p\left(\mathbb{F}-v_{\mathbb{A}}(X)\right)$ for $X \in \operatorname{Lie} \mathcal{G}$. As $c: C \rightarrow M$ is $\mathcal{G}$-invariant, we can integrate $\left(c \times \operatorname{id}_{M}\right)^{*} p_{\mathcal{G}}^{\mathbb{A}} \in \Omega_{\mathcal{G}}^{2 r}(C \times N)$ 
over $C$ to obtain $\varpi_{c}=\int_{c} p_{\mathcal{G}}^{\mathbb{A}} \in \Omega_{\mathcal{G}}^{2}(N)$. We have $\varpi_{c}=\sigma_{c}+\mu_{c}$, with $\sigma_{c}=\int_{c} p(\mathbb{F})$ and $\mu_{c}$ a co-moment map for $\sigma_{c}$ given by $\mu_{c}(X)=-r \int_{c} p\left(v_{\mathbb{A}}(X), \mathbb{F},{ }^{(r-1)}, \mathbb{F}\right)$ for $X \in \mathrm{Lie} \mathcal{G}$.

As it is commented in Introduction, the equivariant prequantization bundles are given in terms of a background connection. Let $A_{0}$ be a connection on $P \rightarrow M$ (we call $A_{0}$ a background connection). If $\mathrm{pr}_{1}: P \times N \rightarrow P$ denotes the projection, then $\mathbb{A}$ and $\bar{A}_{0}=$ $\operatorname{pr}_{1}^{*} A_{0}$ are connections on the same bundle $P \times N \rightarrow M \times N$, and hence we can define $T p\left(\mathbb{A}, \bar{A}_{0}\right) \in \Omega^{2 r-1}(M \times N)$. The product structure on $M \times N$ induces a bigrading on $\Omega^{k}(M \times N) \simeq \bigoplus_{i=0}^{k} \Omega^{i, k-i}(M \times N)$. We have $p(\mathbb{F})=d T p\left(\mathbb{A}, \bar{A}_{0}\right)+\operatorname{pr}_{1}^{*} p\left(F_{0}\right)$, with $\operatorname{pr}_{1}^{*} p\left(F_{0}\right) \in \Omega^{2 r, 0}(M \times N)$. Hence for any $u: U \rightarrow M$ with $d=\operatorname{dim} U<2 r-1$ we have

$$
\int_{u} p(\mathbb{F})=\int_{u} d T p\left(\mathbb{A}, \bar{A}_{0}\right)=d \int_{u} T p\left(\mathbb{A}, \bar{A}_{0}\right)-(-1)^{d} \int_{\partial u} T p\left(\mathbb{A}, \bar{A}_{0}\right),
$$

where we have used that $\int_{u} \operatorname{pr}_{1}^{*} p\left(F_{0}\right)=0$ as $\operatorname{pr}_{1}^{*} p\left(F_{0}\right) \in \Omega^{2 r, 0}(M \times N)$. In particular, if we define $\rho_{c}=\int_{c} \operatorname{Tp}\left(\mathbb{A}, \bar{A}_{0}\right) \in \Omega^{1}(N)$, then by using Eq. (3) and that $\partial c=0$ we obtain

$$
\sigma_{c}=\int_{c} p(\mathbb{F})=d \rho_{c} .
$$

\subsection{Discrete group}

Assume that $\mathcal{G}$ is a discrete group. Let $E$ be a manifold in which $\mathcal{G}$ acts and such that the following condition is satisfied:

(*) $E$ is connected and simply connected and $\pi: N \times E \rightarrow(N \times E) / \mathcal{G}$ is a principal $\mathcal{G}$-bundle.

For example, we can take $E=\mathbf{E} \mathcal{G}$ or another simpler manifold. We define $N^{\prime}=N \times E$ and we denote by $q: M \times N^{\prime} \rightarrow M \times N$ and $\bar{q}: \mathbb{P}^{\prime}=\mathbb{P} \times E \rightarrow \mathbb{P}$ the projections, which are $\mathcal{G}$-equivariant maps. Hence $\bar{q}^{*} \mathbb{A}$ is a $\mathcal{G}$-invariant connection on $\mathbb{P}^{\prime} \rightarrow M \times N^{\prime}$, and it projects onto a connection $\underline{\mathbb{A}}$ on the quotient principal $G$-bundle $\mathbb{P}^{\prime} / \mathcal{G} \rightarrow\left(M \times N^{\prime}\right) / \mathcal{G}$. We denote by $\mathbb{F}$ the curvature of $\underline{\mathbb{A}}$. Given $(p, \Upsilon) \in \mathcal{I}_{\mathbb{Z}}^{r}(G)$, we have the Chern-Simons character $\chi_{\mathbb{A}} \in \hat{H}^{2 r}\left(\left(M \times N^{\prime}\right) / \mathcal{G}\right)$. As $c: C \rightarrow M$ is a $\mathcal{G}$-equivariant map, it induces a map

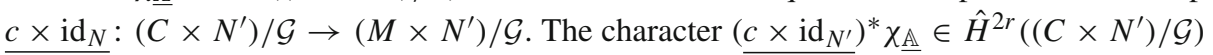
can be integrated over the fiber of $\left(C \times N^{\prime}\right) / \mathcal{G} \rightarrow N^{\prime} / \mathcal{G}$, and we obtain a differential character $\xi_{c}=\int_{c} \chi_{\underline{\mathbb{A}}}=\int_{C}\left(c \times \operatorname{id}_{N}\right)^{*} \chi_{\mathbb{A}} \in \hat{H}^{2}\left(N^{\prime} / \mathcal{G}\right)$. We have $\operatorname{curv}\left(\xi_{c}\right)=\int_{c} \operatorname{curv}\left(\chi_{\underline{\mathbb{A}}}\right)=\int_{c} p(\underline{\mathbb{F}})$ and $\operatorname{char}\left(\bar{\xi}_{c}\right)=\int_{c} \overline{\operatorname{char}\left(\chi_{\underline{\mathbb{A}}}\right)}=\int_{c} \Upsilon_{\mathbb{P}^{\prime} / \mathcal{G}}$.

If $A_{0}$ is a background connection, by Eq. (4) we have $\pi^{*}\left(\int_{c} p(\underline{\mathbb{F}})\right)=\int_{c} p\left(q^{*} \mathbb{F}\right)=$ $q^{*} \int_{c} p(\mathbb{F})=d\left(q^{*} \rho_{c}\right)$. By applying Proposition 3 with $\lambda=q^{*} \rho_{c}$, we obtain a cocycle $\bar{\alpha}_{c}: \mathcal{G} \times N \times E \rightarrow \mathbb{R} / \mathbb{Z}$. Precisely, if $\phi \in \mathcal{G}, \gamma$ is a curve on $N$ joining $x$ and $\phi x$ and $\gamma^{\prime}$ is a curve on $E$ joining $e$ and $\phi e$ we have

$$
\bar{\alpha}_{\phi}(x, e)=\int_{\gamma \times \gamma^{\prime}} q^{*} \rho_{c}-\xi_{c}\left(\pi \circ\left(\gamma \times \gamma^{\prime}\right)\right)=\int_{\gamma} \rho_{c}-\xi_{c}\left(\pi \circ\left(\gamma \times \gamma^{\prime}\right)\right) .
$$

Remark 13 Note that by Lemma $5 \bar{\alpha}_{\phi}$ is differentiable and $d \bar{\alpha}_{\phi}=\phi^{*} q^{*} \rho_{c}-q^{*} \rho_{c}=$ $q^{*}\left(\phi^{*} \rho_{c}-\rho_{c}\right)$.

\section{Lemma 14 We have}

(a) $\bar{\alpha}_{\phi}(x, e)$ does not depend on $e \in E$, and hence $\bar{\alpha}=q^{*} \alpha$ for a cocycle $\alpha: \mathcal{G} \times N \rightarrow \mathbb{R} / \mathbb{Z}$. Furthermore, $\alpha$ satisfies $\alpha_{\phi_{2} \phi_{1}}(x)=\alpha_{\phi_{1}}(x)+\alpha_{\phi_{2}}\left(\phi_{1} x\right)$ and $d \alpha_{\phi}=\phi^{*} \rho_{c}-\rho_{c}$ for $\phi$, $\phi_{1}, \phi_{2} \in \mathcal{G}$. 
(b) $\alpha_{c}$ does not depend on the chosen manifold $E$.

Proof (a) By Lemma 5, if $e^{\prime}$ is another point on $E$ and $\gamma_{e e^{\prime}}$ a curve on $E$ joining $e$ and $e^{\prime}$ (it exists as $E$ is connected) we have $\alpha_{\phi}\left(x, e^{\prime}\right)=\alpha_{\phi}(x, e)+\int_{\{x\} \times \gamma_{e e^{\prime}}}\left(\phi^{*} q^{*} \rho_{c}-q^{*} \rho_{c}\right)$. But $\int_{\{x\} \times \gamma_{e e^{\prime}}}\left(\phi^{*} q^{*} \rho_{c}-q^{*} \rho_{c}\right)=\int_{\{x\} \times \gamma_{e e^{\prime}}} q^{*}\left(\phi^{*} \rho_{c}-\rho_{c}\right)=0$, and hence, $\alpha_{\phi}\left(x, e^{\prime}\right)=$ $\alpha_{\phi}(x, e)$.

(b) Let $E_{1}, E_{2}$ be two manifolds satisfying condition (*). Then $E_{3}=E_{1} \times E_{2}$ also satisfies (*). We define $Q_{i}:\left(P \times N \times E_{3}\right) / \mathcal{G} \rightarrow\left(P \times N \times E_{i}\right) / \mathcal{G}$, and $q_{i}:(N \times E) / \mathcal{G} \rightarrow$ $\left(N \times E_{i}\right) / \mathcal{G}, i=1,2$. We have $Q_{1}^{*} \bar{q}_{1}^{*} \mathbb{A}=Q_{2}^{*} \bar{q}_{2}^{*} \mathbb{A}=\bar{q}_{3}^{*} \mathbb{A}$, and by using Proposition 12 we obtain $q_{1}^{*} \xi_{c}^{1}=q_{2}^{*} \xi_{c}^{2}=\xi_{c}^{3}$.

If $\phi \in \mathcal{G}, \gamma$ is a curve on $N$ joining $x$ and $\phi x$, and $\gamma_{i}$ is a curve on $E_{i}$ joining $e_{i}$ and $\phi e_{i}$, we have $\xi_{c}^{3}\left(\pi_{3} \circ\left(\gamma \times \gamma_{1} \times \gamma_{2}\right)\right)=\xi_{c}^{i}\left(q_{i} \circ \pi_{3} \circ\left(\gamma \times \gamma_{1} \times \gamma_{2}\right)\right)=\xi_{c}^{i}\left(\pi_{i} \circ\left(\gamma \times \gamma_{i}\right)\right)$ for $i=1,2$, and by the definition of $\bar{\alpha}_{i}$ and a) we have $\left(\alpha_{1}\right)_{\phi}(x)=\left(\bar{\alpha}_{1}\right)_{\phi}\left(x, e_{1}\right)=\left(\bar{\alpha}_{3}\right)_{\phi}\left(x, e_{1}, e_{2}\right)=$ $\left(\bar{\alpha}_{2}\right)_{\phi}\left(x, e_{2}\right)=\left(\alpha_{2}\right)_{\phi}(x)$.

The cocycle $\alpha: \mathcal{G} \times N \rightarrow \mathbb{R} / \mathbb{Z}$ defines an action of $\mathcal{G}$ on $\mathcal{U}_{c}=N \times U(1)$ by $U(1)$ bundle automorphisms $\Phi_{\phi}(x, u)=\left(\phi x, \exp \left(2 \pi i \alpha_{\phi}(x)\right) \cdot u\right)$ and the connection form $\Xi_{c}=$ $\theta-2 \pi i \rho_{c}$ is $\mathcal{G}$-invariant.

Hence, for any action of a discrete group $\mathcal{G}$ on $N$ we have the following

Proposition 15 Let $A_{0}$ be a background connection on $P \rightarrow M$. Then there exists a lift of the action of $\mathcal{G}$ on $N$ to an action on $\mathcal{U}_{c}=N \times U(1)$ by $U(1)$-bundle automorphisms such that $\Xi_{c}=\theta-2 \pi i \rho_{c} \in \Omega^{1}(N \times U(1), i \mathbb{R})$ is $\mathcal{G}$-invariant.

We recall that a $\mathcal{G}$-equivariant section of $\mathcal{U}_{c} \rightarrow N$ is determined by a map $S: N \rightarrow U(1)$ $S(x)=\exp (2 \pi i \cdot s(x))$ where $s: N \rightarrow \mathbb{R} / \mathbb{Z}$ satisfies $\alpha_{\phi}(x)=s(\phi x)-s(x)$. The following result shows that our bundle generalizes the Chern-Simons line

Proposition 16 If $c=\partial u$ for a $\mathcal{G}$-equivariant map $u: U \rightarrow M$ and we define $s_{u}=$ $-\int_{u} T p\left(\mathbb{A}, \bar{A}_{0}\right) \in \Omega^{0}(N)$, then $\alpha_{\phi}(x)=s_{u}(\phi x)-s_{u}(x)$. Hence $S_{u}=\exp \left(2 \pi i \cdot s_{u}\right)$ determines a $\mathcal{G}$-equivariant section of $\mathcal{U}_{c} \rightarrow N$.

Furthermore, we have $\nabla^{\Xi_{c}} S_{u}=-2 \pi i \sigma_{u} \cdot S_{u}$, where $\sigma_{u}=\int_{u} p(\mathbb{F})$.

Proof If we define $\sigma_{u}=\int_{u} p(\mathbb{F})$ then $d \sigma_{u}=\int_{u} d(p(\mathbb{F}))+\int_{\partial u} p(\mathbb{F})=\sigma_{c}$. Moreover by Eqs. (3) and (4) we have $\sigma_{u}=\int_{u} p(\mathbb{F})=d \int_{u} T p\left(\mathbb{A}, \bar{A}_{0}\right)+\int_{\partial u} T p\left(\mathbb{A}, \bar{A}_{0}\right)=-d s_{u}+\rho_{c}$. For a curve $\gamma$ joining $x$ and $\phi x$, and $\gamma^{\prime}$ a curve on $E$ joining $e$ and $\phi e$, using the preceding equations and Eq. (2) we have

$$
\begin{aligned}
\alpha_{\phi}(x)=\int_{\gamma} \rho_{c} & -\left(\int_{\partial u} \chi_{\mathbb{A}}\right)\left(\pi \circ\left(\gamma \times \gamma^{\prime}\right)\right)=\int_{\gamma}\left(\sigma_{u}+d s_{u}\right)-\int_{\gamma \times \gamma^{\prime}} q^{*} \int_{u} p(\mathbb{F}) \\
& =\int_{\gamma}\left(\sigma_{u}+d s_{u}\right)-\int_{\gamma} \sigma_{u}=\int_{\gamma} d s_{u}=\int_{\partial \gamma} s_{u}=s_{u}(\phi x)-s_{u}(x) .
\end{aligned}
$$

Finally we have $\nabla^{\Xi_{c}} S_{u}=d S_{u}-2 \pi i \rho_{c} \cdot S_{u}=2 \pi i d s_{u} \cdot S_{u}-2 \pi i \rho_{c} \cdot S_{u}=-2 \pi i \sigma_{u} \cdot S_{u}$.

Remark 17 We can also consider $\exp \left(2 \pi i \cdot \int_{u} T p\left(\mathbb{A}, \bar{A}_{0}\right)\right)$ as a section of the inverse bundle $\mathcal{U}_{c}^{-1}$ as it is done in [14].

Let $\mathcal{H}$ be another discrete group and let $h: \mathcal{H} \rightarrow \mathcal{G}$ be a group homomorphism. Then $h$ induces actions of the group $\mathcal{H}$ on $N$ and $P$ that satisfies conditions a), b) and c). If $\phi \in \mathcal{H}$ we denote by $\alpha_{\phi}^{\mathcal{H}}$ and $\Phi_{\phi}^{\mathcal{H}}$ the cocycle and action determined by the group $\mathcal{H}$ 
Proposition 18 We have $\alpha_{\phi}^{\mathcal{H}}=\alpha_{h(\phi)}^{\mathcal{G}}$ and $\Phi_{\phi}^{\mathcal{H}}=\Phi_{h(\phi)}^{\mathcal{G}}$ for any $\phi \in \mathcal{H}$.

Proof We define $N_{1}=N \times E \mathcal{G}, N_{2}=N \times E \mathcal{G} \times E \mathcal{H}$, and the projections $\bar{q}_{1}: P \times N_{1} \rightarrow$ $P \times N, \bar{q}_{2}: P \times N_{2} \rightarrow P \times N$. Then $h$ induces actions of $\mathcal{H}$ on $P, N$ and $E \mathcal{G}$. We denote by $\underline{\mathbb{A}}_{\mathcal{G}} \in \Omega^{1}\left(\left(P \times N_{1}\right) / \mathcal{G}, \mathfrak{g}\right)$ and $\underline{\mathbb{A}}_{\mathcal{H}} \in \Omega^{1}\left(\left(P \times N_{2}\right) / \mathcal{H}, \mathfrak{g}\right)$ the projections of the connections $\bar{q}_{1}^{*} \mathbb{A}$ and $\bar{q}_{2}^{*} \mathbb{A}$, and by $\xi_{c}^{\mathcal{H}}=\int_{c} \chi_{\mathbb{A}_{\mathcal{H}}} \in \hat{H}^{2}\left(N_{2} / \mathcal{H}\right)$ and $\xi_{c}^{\mathcal{G}}=\int_{c} \chi_{\mathbb{A}_{\mathcal{G}}} \in \hat{H}^{2}\left(N_{1} / \mathcal{G}\right)$ the integrated Chern-Simons characters.

We define a map $\bar{Z}:\left(P \times N_{2}\right) / \mathcal{H} \rightarrow\left(P \times N_{1}\right) / \mathcal{G}$ by $\bar{Z}\left(\left[y, x, e_{1}, e_{2}\right]_{\mathcal{H}}\right)=\left[y, x, e_{1}\right]_{\mathcal{G}}$, and we have $\underline{\mathbb{A}}_{\mathcal{H}}=\bar{Z}^{*}\left(\underline{\mathbb{A}}_{\mathcal{G}}\right)$. In a similar way, we define the maps $Z:\left(M \times N_{2}\right) / \mathcal{H} \rightarrow$ $\left(M \times N_{1}\right) / \mathcal{G}$ and $z: N_{2} / \mathcal{H} \rightarrow N_{1} / \mathcal{G}$. Thus, $Z$ determines a morphism of bundles with fiber $M$ satisfying the conditions of Proposition 12, and we conclude that $\xi_{c}^{\mathcal{H}}=z^{*} \xi_{c}^{\mathcal{G}}$.

Let $\gamma$ be a curve on $N$ joining $x$ and $\phi \cdot x=h(\phi) \cdot x, \gamma_{1}$ a curve on $E \mathcal{G}$ joining $e_{1}$ and $h(\phi) \cdot e_{1}$ and $\gamma_{2}$ a curve on $E \mathcal{H}$ joining $e_{2}$ and $\phi \cdot e_{2}$, and let $\vec{\gamma}_{1}=\gamma \times \gamma_{1} \cdot \vec{\gamma}_{2}=\gamma \times \gamma_{1} \times \gamma_{2}$. By Lemma 14, we have $\alpha_{\phi}^{\mathcal{H}}(x)=\int_{\gamma} \rho_{c}-\xi_{c}^{\mathcal{H}}\left(\pi_{\mathcal{H}} \circ \vec{\gamma}_{2}\right)$ and $\alpha_{h(\phi)}^{\mathcal{G}}(x)=\int_{\gamma} \rho_{c}-\xi_{c}^{\mathcal{G}}\left(\pi_{\mathcal{G}} \circ \vec{\gamma}_{1}\right)$, where $\pi_{\mathcal{G}}: N_{1} \rightarrow N_{1} / \mathcal{G}$, and $\pi_{\mathcal{H}}: N_{2} \rightarrow N_{2} / \mathcal{H}$ are the projections. We have $\xi_{c}^{\mathcal{H}}\left(\pi_{\mathcal{H}} \circ \vec{\gamma}_{2}\right)=$ $z^{*} \xi_{c}^{\mathcal{G}}\left(\pi_{\mathcal{H}} \circ \vec{\gamma}_{2}\right)=\xi_{c}^{\mathcal{G}}\left(z \circ \pi_{\mathcal{H}} \circ \vec{\gamma}_{2}\right)=\xi_{c}^{\mathcal{G}}\left(\pi_{\mathcal{G}} \circ \vec{\gamma}_{1}\right)$, and hence $\alpha_{\phi}^{\mathcal{H}}(x)=\alpha_{h(\phi)}^{\mathcal{G}}(x)$.

\subsection{General group}

In this section, we give a definition of the prequantization bundle valid for arbitrary Lie groups. The definition for discrete groups cannot be generalized directly to Lie groups because the connection $\bar{q}^{*} \mathbb{A}$ is not necessarily projectable to the quotient $(P \times N \times E) / \mathcal{G}$. As commented in Introduction, it is possible to obtain a connection on the quotient space by using an auxiliary connection $\mathfrak{A}$ on the principal $\mathcal{G}$-bundle $N \times E \rightarrow(N \times E) / \mathcal{G}$. As this could be problematic for Fréchet Lie groups, we define the lift of the action of any element $\phi \in \mathcal{G}$ by using the results for discrete groups.

Given $\phi \in \mathcal{G}$, the homomorphism $\mathbb{Z} \rightarrow \mathcal{G} n \mapsto \phi^{n}$ determines actions of the group $\mathbb{Z}$ on $P$ and $N$. We apply the results of Sect. 6.1 to the discrete group $\mathbb{Z}$, and we obtain a cocycle $\alpha_{\phi}: N \rightarrow \mathbb{R} / \mathbb{Z}$ and a lifted action $\Phi_{\phi}: N \times U(1) \rightarrow N \times U(1)$ by $U$ (1)-bundle automorphisms that leaves invariant the connection $\Xi_{c}=\theta-2 \pi i \rho_{c}$. Let us compute $\alpha_{\phi}$ explicitly. We consider the universal $\mathbb{Z}$-bundle $\mathbf{E} \mathbb{Z}=\mathbb{R} \rightarrow \mathbb{Z}$, and the products $\bar{q}: P \times$ $N \times \mathbb{R} \rightarrow P \times N, q: M \times N \times \mathbb{R} \rightarrow M \times N$. The connection $\bar{q}^{*} \mathbb{A}$ is $\mathbb{Z}$-invariant and hence projects onto a connection $\underline{\mathbb{A}}_{\phi}$ on the principal $G$-bundle $(P \times N \times \mathbb{R}) / \mathbb{Z} \rightarrow(M \times N \times \mathbb{R}) / \mathbb{Z}$. Given $(p, \Upsilon) \in \mathcal{I}_{\mathbb{Z}}(G)$, we have the Chern-Simons differential character $\chi_{\mathbb{A}_{\phi}} \in \hat{H}^{2 r}((M \times$ $N \times \mathbb{R})(\mathbb{Z})$, and by integrating over $c$ we obtain the integrated Chern-Simons character $\xi_{c}^{\phi}=\int_{c} \chi_{\mathbb{A}_{\phi}} \in \hat{H}^{2}((N \times \mathbb{R}) / \mathbb{Z})$. If $\gamma$ is any curve on $N$ joining $x$ and $\phi x$, we define $\vec{\gamma}: I \rightarrow N \times \mathbb{R}, \vec{\gamma}(s)=(\gamma(s), s)$ and we have

$$
\alpha_{\phi}(x)=\int_{\vec{\gamma}} q^{*} \rho_{c}-\xi_{c}^{\phi}\left(\pi_{\phi} \circ \vec{\gamma}\right)=\int_{\gamma} \rho_{c}-\xi_{c}^{\phi}\left(\pi_{\phi} \circ \vec{\gamma}\right),
$$

where $\pi_{\phi}: N \times \mathbb{R} \rightarrow(N \times \mathbb{R}) / \mathbb{Z}$ denotes the projection.

Note that $(N \times \mathbb{R}) / \mathbb{Z}$ can be identified with $(N \times I) / \sim_{\phi}$ where the equivalence relation is defined by $(x, 0) \sim_{\phi}(\phi x, 1)$. With this identification, we have $\pi_{\phi} \circ \vec{\gamma}(s)=[\gamma(s), s]_{\phi}$, which is a closed curve as we have $(\gamma(1), 1)=(\phi x, 1) \sim_{\phi}(x, 0)=(\gamma(0), 0)$.

The map $\Phi_{\phi}: N \times U(1) \rightarrow N \times U(1), \Phi_{\phi}(x, u)=\left(\phi x, \exp \left(2 \pi i \alpha_{\phi}(x)\right) \cdot u\right)$ defines a group action of $\mathcal{G}$ on $N \times U(1)$ as we have the following

Proposition 19 For any $\phi_{1}, \phi_{2} \in \mathcal{G}$, we have $\alpha_{\phi_{2} \phi_{1}}(x)=\alpha_{\phi_{1}}(x)+\alpha_{\phi_{2}}\left(\phi_{1} x\right)$, and hence $\Phi_{\phi_{2} \phi_{1}}=\Phi_{\phi_{2}} \circ \Phi_{\phi_{1}}$. 
Proof Given $\phi_{1}, \phi_{2} \in \mathcal{G}$ we consider the free group $F_{2}\left[x_{1}, x_{2}\right]$ generated by two elements $x_{1}, x_{2}$. The assignment $x_{1} \mapsto \phi_{1}, x_{2} \mapsto \phi_{2}$ defines and action of the discrete group $F_{2}\left[x_{1}, x_{2}\right]$ on $P \times N$. We have two homomorphisms $h_{i}: \mathbb{Z} \rightarrow F_{2}\left[x_{1}, x_{2}\right]$ determined by setting $h_{i}(n)=$ $x_{i}^{n}$. By applying Lemma 14 and Proposition 18, we obtain $\alpha_{\phi_{2} \phi_{1}}(x)=\alpha_{\phi_{1}}(x)+\alpha_{\phi_{2}}\left(\phi_{1} x\right)$ for any $x \in N$.

Lemma 20 If $\phi_{t} \in \mathcal{G}, t \in(-\varepsilon, \varepsilon)$ is a local 1-parametric subgroup of $\mathcal{G}$ with $\dot{\phi}_{t}=X \in$ Lie $\mathcal{G}$, then we have $\left.\frac{d \alpha_{\phi_{t}}}{d t}\right|_{t=0}=\rho_{c}\left(X_{N}\right)+\mu_{c}(X)$.

Proof Given $x \in N$, we define $\gamma(s)=\phi_{s} x$ and for $t \in(-\varepsilon, \varepsilon), \gamma^{t}(s)=\gamma(t s)$ and $\vec{\gamma}^{t}(s)=\left(\gamma^{t}(s), s\right)$. We have $\vec{\gamma}^{t}(0)=\left(\phi_{0} x, 0\right)=(x, 0)$ and $\vec{\gamma}^{t}(1)=\left(\phi_{t} x, 1\right) \sim_{\phi_{t}}(x, 0)$. By definition, we have $\alpha_{\phi_{t}}(x)=\int_{\gamma^{t}} \rho_{c}-\xi_{c}^{\phi_{t}}\left(\pi_{\phi_{t}} \circ \vec{\gamma}^{t}\right)$.

The derivative of the first term is easy to compute, as we have $\int_{\gamma^{t}} \rho_{c}=\int_{0}^{1} \rho_{c}\left(\dot{\gamma}^{t}(s)\right) d s=$ $\int_{0}^{1} \rho_{c}(\dot{\gamma}(t s)) t d s \underset{z=t s}{=} \int_{0}^{t} \rho_{c}(\dot{\gamma}(z)) d z$ and hence $\left.\frac{\mathrm{d}}{\mathrm{d} t} \int_{\gamma^{t}} \rho_{c}\right|_{t=0}=\rho_{c}\left(X_{N}(x)\right)$.

Next we compute the derivative of the second term. We denote by $N_{t}$ the manifold $N \times \mathbb{R}$ with the action of $\mathbb{Z}$ determined by $n \cdot(x, s)=\left(\left(\phi_{t}\right)^{n} x, s+n\right)$. The maps $w_{t}: N_{0} \rightarrow N_{t}$, $w_{t}(x, s)=\left(\phi_{t s} x, s\right), W_{t}: P \times N_{0} \rightarrow P \times N_{t}, W_{t}(y, x, s)=\left(\phi_{t s} y, \phi_{t s} x, s\right)$ for $y \in P, x \in$ $N$ and $s \in \mathbb{R}$ are $\mathbb{Z}$-equivariant. We denote by $\underline{w}_{t}: N_{0} / \mathbb{Z} \rightarrow N_{t} / \mathbb{Z}$ and $\underline{W}_{t}:\left(P \times N_{0}\right) / \mathbb{Z} \rightarrow$ $\left(P \times N_{t}\right) / \mathbb{Z}$ the induced maps. We define the connections $A_{t}=W_{t}^{*} q^{*} \mathbb{A}$, and we have $A_{0}=q^{*} \mathbb{A}$ and $\dot{A}_{0}=\left.\frac{\mathrm{d}}{\mathrm{d} t}\right|_{t=0}\left(W_{t}^{*} q^{*} \mathbb{A}\right)=L_{Y} q^{*} \mathbb{A}$, where $Y=\left.\frac{\mathrm{d} W_{t}}{\mathrm{~d} t}\right|_{t=0}=s X_{P \times N}$. As $q^{*} \mathbb{A}$ is $\mathcal{G}$-invariant, we have $\dot{A}_{0}=L_{Y} q^{*} \mathbb{A}=s q^{*}\left(L_{X_{P \times N}} \mathbb{A}\right)+q^{*}\left(\iota_{X_{P \times N}} \mathbb{A}\right) d s=\left(q^{*} v_{\mathbb{A}}(X)\right) d s$.

We denote by $\underline{A}_{t}$ the projection of $A_{t}$ on $\left(P \times N_{0}\right) / \mathbb{Z}$ and we define $\zeta_{c}^{t}=\int_{c} \chi_{\underline{A}_{t}} \in$ $\hat{H}^{2}\left(N_{0} / \mathbb{Z}\right)$. In each of the cases $\left.\left.\mathrm{c}_{1}\right), \mathrm{c}_{2}\right)$ and $\left.\mathrm{c}_{3}\right)$ by using Proposition 12 , we obtain $\zeta_{c}^{t}=$ $\underline{w}_{t}^{*}\left(\xi_{c}^{\phi_{t}}\right)$.

If $\varrho_{x}: I \rightarrow N_{0}$ is the curve $\varrho_{x}(s)=(x, s)$, then we have $\pi_{\phi_{t}} \circ \vec{\gamma}^{t}=\underline{w}_{t} \circ \pi_{\phi_{0}} \circ \varrho_{x}$ and hence $\xi_{c}^{\phi_{t}}\left(\pi_{\phi_{t}} \circ \vec{\gamma}^{t}\right)=\xi_{c}^{\phi_{t}}\left(\underline{w}_{t} \circ \pi_{\phi_{0}} \circ \varrho_{x}\right)=\zeta_{c}^{t}\left(\pi_{\phi_{0}} \circ \varrho_{x}\right)$. By using Lemma 10, we conclude that

$$
\begin{aligned}
\left.\frac{\mathrm{d}}{\mathrm{d} t} \xi_{c}^{\phi_{t}}\left(\pi_{\phi_{t}} \circ \vec{\gamma}^{t}\right)\right|_{t=0} & =\left.\frac{\mathrm{d}}{\mathrm{d} t} \zeta_{c}^{t}\left(\pi_{\phi_{0}} \circ \varrho_{x}\right)\right|_{t=0}=r \int_{\varrho_{x}} \int_{c} p\left(\dot{A}_{0}, q^{*} \mathbb{F},(r-1), q^{*} \mathbb{F}\right) \\
& =\left(r \int_{c} p\left(v_{\mathbb{A}}(X), \mathbb{F}, \stackrel{(r-1)}{\cdots}, \mathbb{F}\right)\right)_{x}\left(\int_{\varrho_{x}} d s\right)=-\mu_{c}(X)_{x},
\end{aligned}
$$

and the result follows.

As a consequence of the preceding Lemma, we have the following

Proposition 21 For $X \in \operatorname{Lie} \mathcal{G}$, we have $X_{\mathcal{U}_{c}}=X_{N}+2 \pi i\left(\rho_{c}\left(X_{N}\right)+\mu_{c}(X)\right) \partial_{\theta}$.

We have curv $\Xi_{c}=d \rho_{c}=\sigma_{c}$ and $\iota_{X_{\mathcal{U}_{c}}} \Xi_{c}=2 \pi i \mu_{c}(X)$. Hence $\operatorname{curv}_{\mathcal{G}}\left(\Xi_{c}\right)=\sigma_{c}+\mu_{c}=$ $\varpi_{c}$. We conclude that $\left(\mathcal{U}_{c}, \Xi_{c}\right)$ is a $\mathcal{G}$-equivariant prequantization bundle of $\left(N, \varpi_{c}\right)$. Hence, we have proved the following

Theorem 22 Let $(p, \Upsilon) \in \mathcal{I}_{\mathbb{Z}}^{r}(G), A_{0}$ be a background connection on $P$ and $c: C \rightarrow M$ a $\mathcal{G}$-invariant map. These data determine an action of $\mathcal{G}$ on $\mathcal{U}_{c}=N \times U(1) \rightarrow N$ by $U(1)$-bundle automorphisms $\Phi_{\phi}(x, u)=\left(\phi x, \exp \left(2 \pi i \alpha_{\phi}(x)\right) \cdot u\right)$ such that the connection $\Xi_{c}=\theta-2 \pi i \rho_{c}$ is $\mathcal{G}$-invariant, and the $\mathcal{G}$-equivariant curvature of $\Xi_{c}$ is $\operatorname{curv}_{\mathcal{G}}\left(\Xi_{c}\right)=\varpi_{c}$.

For every $X \in \mathrm{Lie} \mathcal{G}$, we have $X_{\mathcal{U}_{c}}=X_{N}+2 \pi i\left(\rho_{c}\left(X_{N}\right)+\mu_{c}(X)\right) \partial_{\theta}$. 
If $c=\partial u$ for a $\mathcal{G}$-equivariant map $u: U \rightarrow M$ and $s_{u}=-\int_{u} T p\left(\mathbb{A}, \bar{A}_{0}\right)$, then $\alpha_{\phi}(x)=$ $s_{u}(\phi x)-s_{u}(x)$. Hence $S_{u}=\exp \left(2 \pi i \cdot s_{u}\right)$ determines a $\mathcal{G}$-equivariant section of $\mathcal{U}_{c} \rightarrow N$. Moreover, we have $\nabla^{\Xi_{c}} S_{u}=-2 \pi i \sigma_{u} \cdot S_{u}$, where $\sigma_{u}=\int_{u} p(\mathbb{F}) \in \Omega^{1}(N)$.

Remark 23 In place of the principal $U(1)$-bundle $\mathcal{U}_{c}$, we can consider the $\mathcal{G}$-equivariant Hermitian line bundle $\mathcal{L}_{c}=N \times \mathbb{C} \rightarrow N$ with the action $\Phi_{\phi}(x, z)=\left(\phi x, \exp \left(2 \pi i \alpha_{\phi}(x)\right) \cdot z\right)$ and $\Xi_{c}$ determines a Hermitian connection $\nabla^{\Xi_{c}}$ on this bundle with $\nabla^{\Xi_{c}} f=d f-2 \pi i \rho_{c} \cdot f$ for $f: N \rightarrow \mathbb{C}$.

Let $N_{1}$ be another connected and simply connected manifold on which $\mathcal{G}$ acts. If $g: N_{1} \rightarrow$ $N$ is a $\mathcal{G}$-equivariant map, then $\mathbb{A}_{1}=\left(\operatorname{id}_{P} \times g\right)^{*} \mathbb{A}$ is a $\mathcal{G}$-invariant connection on $P \times N_{1} \rightarrow$ $M \times N_{1}$ and conditions a) b) and c) are satisfied. If $A_{0}$ is a background connection, then we have a cocycle $\alpha_{1}$. The following Proposition can be easily proved.

Proposition 24 We have $\left(\alpha_{1}\right)_{\phi}(x)=\alpha_{\phi}(g(x)),\left(\Phi_{1}\right)_{\phi}(x, u)=\Phi_{\phi}(g(x), u)$ and $\left(\Xi_{1}\right)_{c}=$ $\left(g \times \operatorname{id}_{U(1)}\right)^{*} \Xi_{c}$ for $\phi \in \mathcal{G}, x \in N^{\prime}$ and $u \in U(1)$. In particular, the map $g \times \mathrm{id}_{U(1)}:\left(\mathcal{U}_{1}\right)_{c} \rightarrow$ $\mathcal{U}_{c}$ is $\mathcal{G}$-equivariant.

\subsection{Change of background connection}

The prequantization bundle $\mathcal{U}_{c}$ and the connection $\Xi_{c}$ are defined using a background connection $A_{0}$. If $A_{0}^{\prime}$ is another background connection, then we have $T p\left(\mathbb{A}, \bar{A}_{0}^{\prime}\right)=T p\left(\mathbb{A}_{1}, \bar{A}_{0}\right)+$ $T p\left(\bar{A}_{0}, \bar{A}_{0}^{\prime}\right)+d T p\left(\mathbb{A}, \bar{A}_{0}, \bar{A}_{0}^{\prime}\right)$, with $T p\left(\bar{A}_{0}, \bar{A}_{0}^{\prime}\right)=\operatorname{pr}_{1}^{*} T p\left(A_{0}, A_{0}^{\prime}\right) \in \Omega^{2 r-1,0}(M \times N)$, and hence

$$
\begin{aligned}
& \int_{u} \operatorname{Tp}\left(\mathbb{A}, \bar{A}_{0}^{\prime}\right)=\int_{u} \operatorname{Tp}\left(\mathbb{A}, \bar{A}_{0}\right)+\int_{u} T p\left(\bar{A}_{0}, \bar{A}_{0}^{\prime}\right)+\int_{u} d T p\left(\mathbb{A}, \bar{A}_{0}, \bar{A}_{0}^{\prime}\right) \\
& =\int_{u} T p\left(\mathbb{A}, \bar{A}_{0}\right)+\int_{u} T p\left(\bar{A}_{0}, \bar{A}_{0}^{\prime}\right)+d \int_{u} T p\left(\mathbb{A}, \bar{A}_{0}, \bar{A}_{0}^{\prime}\right)+(-1)^{d} \int_{\partial u} T p\left(\mathbb{A}, \bar{A}_{0}, \bar{A}_{0}^{\prime}\right) .
\end{aligned}
$$

If $d=\operatorname{dim} U<2 r-1$, we have $\int_{u} T p\left(\bar{A}_{0}, \bar{A}_{0}^{\prime}\right)=0$ as $T p\left(\bar{A}_{0}, \bar{A}_{0}^{\prime}\right) \in \Omega^{2 r-1,0}(M \times N)$ and hence

$$
\int_{u} \operatorname{Tp}\left(\mathbb{A}, \bar{A}_{0}^{\prime}\right)=\int_{u} \operatorname{Tp}\left(\mathbb{A}, \bar{A}_{0}\right)+d \int_{u} \operatorname{Tp}\left(\mathbb{A}, \bar{A}_{0}, \bar{A}_{0}^{\prime}\right)+(-1)^{d} \int_{\partial u} \operatorname{Tp}\left(\mathbb{A}, \bar{A}_{0}, \bar{A}_{0}^{\prime}\right) .
$$

Moreover, if $\operatorname{dim} U=2 r-1$ then $\int_{u} T p\left(\mathbb{A}, \bar{A}_{0}, \bar{A}_{0}^{\prime}\right)=0$ and we have

$$
\int_{u} \operatorname{Tp}\left(\mathbb{A}, \bar{A}_{0}^{\prime}\right)=\int_{u} \operatorname{Tp}\left(\mathbb{A}, \bar{A}_{0}\right)+\int_{u} \operatorname{Tp}\left(A_{0}, A_{0}^{\prime}\right)-\int_{\partial u} \operatorname{Tp}\left(\mathbb{A}, \bar{A}_{0}, \bar{A}_{0}^{\prime}\right) .
$$

The next Proposition shows that the action changes under a change of $A_{0}$, but the corresponding prequantization bundles are isomorphic.

Proposition 25 Let $A_{0}^{\prime}$ be another background connection and denote by $\mathcal{U}_{c}^{\prime}$, $\Xi_{c}^{\prime}$ and $\alpha_{c}^{\prime}$ the bundle, connection and action determined by $A_{0}^{\prime}$. If we define $\beta_{c}=\int_{c} \operatorname{Tp}\left(\mathbb{A}, \bar{A}_{0}, \bar{A}_{0}^{\prime}\right) \in$ $\Omega^{0}(N)$, then $\Xi_{c}^{\prime}=\Xi_{c}-2 \pi i d \beta_{c}$ and $\alpha_{\phi}^{\prime}=\alpha_{\phi}+\phi^{*} \beta-\beta$. The map $\Psi: \mathcal{U}_{c} \rightarrow \mathcal{U}_{c}^{\prime} \Psi(x, u)=$ $\left(x, \exp \left(2 \pi i \beta_{c}(x) \cdot u\right)\right.$ is a $\mathcal{G}$-equivariant isomorphism of $U(1)$-bundles and $\Psi^{*}\left(\Xi_{c}^{\prime}\right)=\Xi_{c}$.

Proof It follows easily from the definitions and the equality $\rho_{c}^{\prime}=\rho_{c}+d \beta_{c}$, which is a consequence of Eq. (5). 
Remark 26 We interpret this result in the following way. The pair $(p, \Upsilon) \in I_{\mathbb{Z}}^{r}(G)$ and $c: C \rightarrow M$ determine a $\mathcal{G}$-equivariant prequantization bundle $\left(\mathcal{U}_{c}, \Xi_{c}\right)$ for $\left(N, \varpi_{c}\right)$, and a background connection $A_{0}$ determines a global trivialization of this bundle. In this sense, the prequantization bundle does not depend on $A_{0}$.

The situation is different for the section associated with the Chern-Simons action, as using Eq. (6) we obtain the following

Proposition 27 If $c=\partial u$ for a $\mathcal{G}$-equivariant map $u: U \rightarrow M$ and $S_{u}$, $S_{u}^{\prime}$ are the sections associated with $A_{0}$ and $A_{0}^{\prime}$, then $\Psi \circ S_{u}=S_{u}^{\prime} \cdot \exp \left(2 \pi i \int_{u} T p\left(A_{0}, A_{0}^{\prime}\right)\right)$.

Hence, the section $S_{u}$ is not intrinsically determined by $(p, \Upsilon) \in \mathcal{I}_{\mathbb{Z}}^{r}(G)$ and $u$. To explain this, note that if $S$ is a section satisfying $\nabla \Xi_{c} S=-2 \pi i \sigma_{u} \cdot S$, any other section satisfying this condition is given by $\exp (i a) S$ for $a \in \mathbb{R}$ constant. The background connection $A_{0}$ determines a constant $a$, and another connection $A_{0}^{\prime}$ determines a different constant $a^{\prime}$ and hence a different section.

\subsection{Change of polynomial and submanifold}

The action of $\mathcal{G}$ on $\mathcal{A} \times U(1)$ is defined by a map $\Phi_{\alpha}(x, u)=\left(\phi x, \exp \left(2 \pi i \alpha_{\phi}(x)\right) \cdot u\right)$ where $\alpha: \mathcal{G} \times N \rightarrow \mathbb{R} / \mathbb{Z}$ satisfies the cocycle condition $\alpha_{\phi_{2} \phi_{1}}(x)=\alpha_{\phi_{1}}(x)+\alpha_{\phi_{2}}\left(\phi_{1} x\right)$. If $\alpha$ and $\alpha^{\prime}$ satisfy the cocycle condition, then it is also satisfied by $-\alpha$ and $\alpha+\alpha^{\prime}$, and $\Phi_{-\alpha}=\Phi_{\alpha}^{-1}$ and $\Phi_{\alpha+\alpha^{\prime}}=\Phi_{\alpha} \cdot \Phi_{\alpha^{\prime}}$. In terms of line bundles, if $\mathcal{L}^{\alpha}$ is the $\mathcal{G}$-equivariant line bundle associated with a cocycle $\alpha$, then $\Phi_{-\alpha}$ corresponds to the dual bundle $\mathcal{L}^{-\alpha}=\left(\mathcal{L}^{\alpha}\right)^{*}$ and $\Phi_{\alpha+\alpha^{\prime}}$ corresponds to the tensor product $\mathcal{L}^{\alpha+\alpha^{\prime}}=\mathcal{L}^{\alpha} \otimes \mathcal{L}^{\alpha^{\prime}}$.

We denote by $\alpha_{c}^{\vec{p}}$ the action determined by $\vec{p}=(p, \Upsilon) \in \mathcal{I}_{\mathbb{Z}}^{r}(G), c: C \rightarrow N$ and by $\left(\mathcal{L}_{c}^{\vec{p}}, \nabla_{c}^{\vec{p}}\right)$ the $\mathcal{G}$-equivariant line bundle and connection determined by them. If $c: C \rightarrow N$, $c^{\prime}: C^{\prime} \rightarrow N$ are two smooth maps we define $-c:-C \rightarrow N$, where $-C$ is the manifold $C$ with the opposite orientation and $c+c^{\prime}: C \sqcup C^{\prime} \rightarrow N$. Then we have $\alpha_{-c}^{\vec{p}}=-\alpha_{c}^{\vec{p}}$, $\alpha_{c+c^{\prime}}^{\vec{p}}=\alpha_{c}^{\vec{p}}+\alpha_{c^{\prime}}^{\vec{p}}$ and also $\rho_{-c}^{\vec{p}}=-\rho_{c}^{\vec{p}}, \rho_{c+c^{\prime}}^{\vec{p}}=\rho_{c}^{\vec{p}}+\rho_{c^{\prime}}^{\vec{p}}$. We conclude that $\left(\mathcal{L}_{-c}^{\vec{p}}, \nabla_{-c}^{\vec{p}}\right)=$ $\left(\left(\mathcal{L}_{c}^{\vec{p}}, \nabla_{c}^{\vec{p}}\right)\right)^{*}$ and $\left(\mathcal{L}_{c+c^{\prime}}^{\vec{p}}, \nabla_{c+c^{\prime}}^{\vec{p}}\right)=\left(\mathcal{L}_{c}^{\vec{p}}, \nabla_{c}^{\vec{p}}\right) \otimes\left(\mathcal{L}_{c^{\prime}}^{\vec{p}}, \nabla_{c^{\prime}}^{\vec{p}}\right)$.

In a similar way if $\vec{p}=(p, \Upsilon), \vec{p}^{\prime}=\left(p^{\prime}, \Upsilon^{\prime}\right) \in \mathcal{I}_{\mathbb{Z}}^{r}(G)$ then we have $\alpha_{c}^{-\vec{p}}=-\alpha_{c}^{\vec{p}}$, $\alpha_{c}^{\vec{p}+\vec{p}^{\prime}}=\alpha_{c}^{\vec{p}}+\alpha_{c}^{\vec{p}^{\prime}}$ and $\rho_{c}^{-\vec{p}}=-\rho_{c}^{\vec{p}}, \rho_{c}^{\vec{p}+\vec{p}^{\prime}}=\rho_{c}^{\vec{p}}+\rho_{c}^{\vec{p}^{\prime}}$. Hence $\left(\mathcal{L}_{c}^{-\vec{p}}, \nabla_{c}^{-\vec{p}}\right)=\left(\left(\mathcal{L}_{c}^{\vec{p}}, \nabla_{c}^{\vec{p}}\right)\right)^{*}$ and $\left(\mathcal{L}_{c}^{\vec{p}+\vec{p}^{\prime}}, \nabla_{c}^{\vec{p}+\vec{p}^{\prime}}\right)=\left(\mathcal{L}_{c}^{\vec{p}}, \nabla_{c}^{\vec{p}}\right) \otimes\left(\mathcal{L}_{c}^{\vec{p}^{\prime}}, \nabla_{c}^{\vec{p}^{\prime}}\right)$.

If $\partial u=c-c^{\prime}$, by Theorem $22 S_{u}=\exp \left(-2 \pi i \cdot \int_{u} T p\left(\mathbb{A}, \bar{A}_{0}\right)\right)$ determines a $\mathcal{G}$-equivariant section of unit norm of $\mathcal{L}_{c-c^{\prime}}^{\vec{p}} \simeq \mathcal{L}_{c}^{\vec{p}} \otimes\left(\mathcal{L}_{c^{\prime}}^{\vec{p}}\right)^{*} \simeq \operatorname{Hom}\left(\mathcal{L}_{c^{\prime}}^{\vec{p}}, \mathcal{L}_{c}^{\vec{p}}\right)$ and hence $\mathcal{L}_{c^{\prime}}^{\vec{p}}$ and $\mathcal{L}_{c}^{\vec{p}}$ are isomorphic as $\mathcal{G}$-equivariant line bundles.

Remark 28 It is important to recall that in the preceding formulas we are using the same background connection $A_{0}$ (i.e., the same trivialization (see Remark 26)) for all the bundles. If we use different connections $A_{0}$ and $A_{0}^{\prime}$ for $c$ and $-c$, we do not have $\mathcal{L}_{c}^{A_{0}}=\left(\mathcal{L}_{-c}^{A_{0}^{\prime}}\right)^{*}$, but we have a pairing $\mathcal{L}_{c}^{A_{0}} \otimes\left(\mathcal{L}_{-c}^{A_{0}^{\prime}}\right)^{*} \rightarrow N \times \mathbb{C}$.

\section{Application to the space of connections}

In this section, the constructions of Sect. 6.2 are applied to the space of connections on a principal $G$-bundle $P \rightarrow M$. First we give the explicit expressions of the forms that appear 
in the prequantization bundle. A background connection is simply an element $A_{0} \in \mathcal{A}$. The 1 -form $\rho_{c}=\int_{c} T p\left(\mathbb{A}, \bar{A}_{0}\right) \in \Omega^{1}(\mathcal{A})$ that determines the connection $\Xi_{c}$ is given by

$$
\left(\rho_{c}\right)_{A}(a)=r(r-1) \int_{c} \int_{0}^{1} p\left(a, A-A_{0}, F_{t}, \stackrel{(r-2)}{\cdots}, F_{t}\right) t \mathrm{~d} t,
$$

with $A_{t}=t A+(1-t) A_{0}$ and $F_{t}$ the curvature of $A_{t}$.

The form $\beta_{c}=\int_{c} T p\left(\mathbb{A}, \bar{A}_{0}, \bar{A}_{0}^{\prime}\right)$ that appears in the change of background connection is simply given by $\beta_{c}(A)=\int_{c} T p\left(A, A_{0}, A_{0}^{\prime}\right)$ (this follows from the tautological definition of $\mathbb{A})$. Finally, if $u: U \rightarrow M$ is a $\mathcal{G}$-invariant map such that $c=\partial u$ then $s_{u}=-\int_{u} T p\left(\mathbb{A}, \bar{A}_{0}\right)$ is given by $s_{u}(A)=-\int_{u} T p\left(A, A_{0}\right)$. Also we have $\left(\sigma_{u}\right)_{A}(a)=r \int_{u} p(a, F, \stackrel{(2 r-1)}{\cdots}, F)$ for $a \in T_{A} \mathcal{A} \simeq \Omega^{1}(M, \operatorname{ad} P)$.

\subsection{Action by gauge transformations}

Now we consider the action of the group $\mathcal{G}=$ Gau $P$ of gauge transformations on $P \rightarrow M$. In this case, as $\mathcal{G}$ does not act on $M$, we can consider any smooth map $c: C \rightarrow M$ with $\operatorname{dim} C=2 r-2$. We summarize the results in the following

Theorem 29 Let $P \rightarrow M$ principal $G$-bundle, $(p, \Upsilon) \in \mathcal{I}_{\mathbb{Z}}^{r}(G)$, $A_{0}$ be a background connection on $P$ and $c: C \rightarrow M$ be a smooth map with $C$ closed, oriented and $\operatorname{dim} C=$ $2 r-2$. If $\mathcal{G}$ acts on $P$ by elements of $\mathrm{Gau} P$, these data determine an action of $\mathcal{G}$ on $\mathcal{U}_{c}=$ $\mathcal{A} \times U(1) \rightarrow \mathcal{A}$ by $U(1)$-bundle automorphisms such that the connection $\Xi_{c}=\theta-2 \pi i \rho_{c}$ is $\mathcal{G}$-invariant and the equivariant curvature of $\Xi_{c}$ is $\varpi_{c}$.

Furthermore, if $c=\partial u$ and $s_{u}(A)=-\int_{u} T p\left(A, A_{0}\right)$, then $\alpha_{\phi}(x)=s_{u}(\phi x)-s_{u}(x)$. Hence $S_{u}=\exp \left(2 \pi i \cdot s_{u}\right)$ determines $\mathcal{G}$-equivariant section of $\mathcal{U}_{c} \rightarrow \mathcal{A}$, and we have $\nabla \Xi_{c} S_{u}=-2 \pi i \sigma_{u} \cdot S_{u}$.

Remark 30 In the classical case of Chern-Simons theory considered in Introduction, any $S U$ (2)-bundle $P$ over a 3-manifold is trivial. Hence, we can take $A_{0}$ the connection corresponding to a global section $u: M \rightarrow P$. Then for $p(X)=\frac{1}{8 \pi^{2}} \operatorname{tr}\left(X^{2}\right)$ we have $s_{M}(A)=-\int_{M} T p\left(A, A_{0}\right)=-\frac{1}{8 \pi^{2}} \int_{M} \operatorname{tr}\left(A \wedge d A+\frac{2}{3} A \wedge A \wedge A\right)$, which coincides with the classical Chern-Simons action.

Remark 31 In [24] the equation $\alpha_{\phi}(A)=s_{u}(\phi A)-s_{u}(A)=-\int_{u} T p\left(\phi A, A_{0}\right)+$ $\int_{u} T p\left(A, A_{0}\right)$ is used to define the action $\alpha_{\phi}$. To do this, it is necessary to express the manifold $c$ as the boundary of another manifold $u$ and to extend the connections on $c$ to $u$. This can be done in dimension two, but this procedure cannot be generalized to higher dimensions.

In [10], a different construction of the bundle provided by theorem 3 is given. If $\mathcal{G}$ is the subgroup of gauge transformations fixing a point of $P$, then $\mathcal{G}$ acts freely on $\mathcal{A}$ and $\mathcal{A} \rightarrow \mathcal{A} / \mathcal{G}$ is a principal $\mathcal{G}$-bundle (see [13]). If $\mathfrak{A}$ is a connection on $\mathcal{A} \rightarrow \mathcal{A} / \mathcal{G}$, we define $\mathbb{A}(\mathfrak{A}) \in \Omega^{1}(P \times \mathcal{A}, \mathfrak{g})$ by $\mathbb{A}(\mathfrak{A})(Y)=\mathbb{A}\left(\left(\mathfrak{A}\left(\left(\mathrm{pr}_{2}\right)_{*} \pi_{*} Y\right)\right)_{P \times \mathcal{A}}\right)$ for $Y \in T(P \times \mathcal{A})$. Then the connection $\mathbb{A}-\mathbb{A}(\mathfrak{A})$ is projectable onto a connection $\underline{\mathfrak{A}}$ on $(P \times \mathcal{A}) / \mathcal{G} \rightarrow M \times \mathcal{A} / \mathcal{G}$. If we set $\lambda_{c}=\rho_{c}+\mu_{c}(\mathfrak{A})$, then we have (see [10]) $d \lambda_{c}=\pi^{*}\left(\int_{c} p\left(F_{\mathfrak{A}}\right)\right)=\operatorname{curv}\left(\int_{c} \chi_{\mathfrak{A}}\right)$. Hence, we can apply Theorem 3 to the character $\int_{c} \chi_{\mathfrak{A}} \in \hat{H}^{2}(\mathcal{A} / \mathcal{G})$, and we obtain a cocycle $a_{\phi}^{\mathfrak{A}}(A)=\int_{\gamma}\left(\rho_{c}+\mu_{c}(\mathfrak{A})\right)-\left(\int_{c} \chi_{\mathfrak{A}}\right)(\pi \circ \gamma)$ that in theory determines another bundle. But this bundle coincides with ours, as we have the following

Proposition 32 We have $a_{\phi}^{\mathfrak{A}}=\alpha_{\phi}$ for any $\phi \in \mathcal{G}$. 
Proof We define the projections $\bar{q}: P \times \mathcal{A} \times \mathbb{R} \rightarrow P \times \mathcal{A}$. The homomorphism $h: \mathbb{Z} \rightarrow \mathcal{G}$ $h(n)=\phi^{n}$ determines an action of $\mathbb{Z}$ on $P$ and $\mathcal{A}$.

Let $\pi_{\mathcal{G}}: P \times \mathcal{A} \rightarrow(P \times \mathcal{A}) / \mathcal{G}$ and $\pi_{\phi}: P \times \mathcal{A} \times \mathbb{R} \rightarrow(P \times \mathcal{A} \times \mathbb{R}) / \mathbb{Z}$ denote the projections. If $\phi \in \mathcal{G}, x \in N, \gamma$ is a curve on $N$ joining $A$ and $\phi A$ and $\vec{\gamma}(s)=(\gamma(s), s)$ by definition we have

$$
\begin{aligned}
\alpha_{\phi}(x) & =\int_{\gamma} \rho_{c}-\int_{c} \chi_{\bar{q}^{*} \mathbb{A}_{\phi}}\left(\pi_{\phi} \circ \vec{\gamma}\right), \\
a_{\phi}^{\mathfrak{A}, \mathcal{G}}(x) & \left.=\int_{\gamma} \rho_{c}+\int_{\gamma} \mu_{c}(\mathfrak{A})\right)-\left(\int_{c} \chi_{\mathfrak{A}}\right)\left(\pi_{\mathcal{G}} \circ \gamma\right) .
\end{aligned}
$$

The connections $\bar{q}^{*} \mathbb{A}$ and $\bar{q}^{*} \pi_{\mathcal{G}}^{*} \mathfrak{A}$ are $\mathbb{Z}$-invariant and project onto connections $\bar{q}^{*} \mathbb{A}$ and $A_{2}=\bar{q}^{*} \pi_{\mathcal{G}}^{*} \underline{A}$ on $(P \times \mathcal{A} \times \mathbb{R}) / \mathbb{Z} \rightarrow(M \times \mathcal{A} \times \mathbb{R}) / \mathbb{Z}$ and by Eq. (1) we have $\int_{c} \chi_{\bar{q}^{*} \mathbb{A}} \overline{\left(\pi_{\phi} \circ \vec{\gamma}\right)}=\int_{c} \chi_{A_{2}}\left(\pi_{\phi} \circ \vec{\gamma}\right)+\int_{\vec{\gamma}} \int_{c} T p\left(\bar{q}^{*} \mathbb{A}, \bar{q}^{*} \pi_{\mathcal{G}}^{*} \mathfrak{A}\right)$. But $\int_{\vec{\gamma}} \int_{c} T p\left(\bar{q}^{*} \mathbb{A}, \bar{q}^{*} \pi_{\mathcal{G}}^{*} \mathfrak{A}\right)=$ $\int_{\gamma} \overline{\int_{c} T} p\left(\mathbb{A}, \pi_{\mathcal{G}}^{*} \mathfrak{A}\right)=\int_{\gamma} \int_{c} T p\left(\mathbb{A}, \pi_{\mathcal{G}}^{*} \mathfrak{A}\right)^{2 r-2,1}$, where the bigradation is the one induced by the product structure on $M \times \mathcal{A}$. We have $T p\left(\mathbb{A}, \pi_{\mathcal{G}}^{*} \mathfrak{A}\right)^{2 r-2,1}=r \int_{0}^{1} p\left(\mathbb{A}(\mathfrak{A}), F_{t}, \ldots, F_{t}\right)$ with $F_{t}=\mathbb{F}+t d_{\mathbb{A}} \mathbb{A}(\mathfrak{A})+\frac{t^{2}}{2}[\mathbb{A}(\mathfrak{A}), \mathbb{A}(\mathfrak{A})]$. As $\mathfrak{A}$ comes from a connection on $\mathcal{A} \rightarrow \mathcal{A} / \mathcal{G}$, we have $F_{t}^{2,0}=\mathbb{F}^{2,0}$ and hence $\int_{\gamma} \int_{c} T p\left(\mathbb{A}, \pi_{\mathcal{G}}^{*} \mathfrak{A}\right)=r \int_{\gamma} \int_{c} p(\mathbb{A}(\mathfrak{A}), \mathbb{F}, \ldots, \mathbb{F})=-\int_{\gamma} \mu_{c}(\mathfrak{A})$. We conclude that we have $\alpha_{\phi}(x)=\int_{\gamma} \rho_{c}+\int_{\gamma} \mu_{c}(\mathfrak{A})-\int_{c} \chi_{A_{2}}\left(\pi_{\phi} \circ \vec{\gamma}\right)$.

Hence we should prove that $\int_{c} \chi_{A_{2}}\left(\pi_{\phi} \circ \vec{\gamma}\right)=\int_{c} \chi_{\mathfrak{A}}\left(\pi_{\mathcal{G}} \circ \gamma\right)$. This result follows in a similar way as in the proof of Proposition 18. If $\bar{Z}:(P \times \mathcal{A} \times \mathbb{R}) / \mathbb{Z} \rightarrow(P \times \mathcal{A}) / \mathcal{G}$ and $z:(\mathcal{A} \times \mathbb{R}) / \mathbb{Z} \rightarrow \mathcal{A} / \mathcal{G}$ are the natural maps, then we have $A_{2}=\bar{Z}^{*} \mathfrak{A}$ and $\int_{c} \chi_{A_{2}}=z^{*} \int_{c} \chi_{\mathfrak{A}}$. Hence $\left(\int_{c} \chi_{A_{2}}\right)\left(\pi_{\phi} \circ \vec{\gamma}\right)=\left(\int_{c} \chi_{\mathfrak{A}}\right)\left(z \circ \pi_{\phi} \circ \vec{\gamma}\right)=\left(\int_{c} \chi_{\mathfrak{A}}\right)\left(\pi_{\mathcal{G}} \circ \gamma\right)$.

In particular, the action $a_{\phi}^{\mathfrak{A}}$ does not depend on the connection $\mathfrak{A}$ chosen on $\mathcal{A} \rightarrow \mathcal{A} / \mathcal{G}$.

\subsection{Restriction to the moduli space of irreducible flat connections}

We denote by $\widetilde{\mathcal{A}}$ the space of irreducible connections. Although Gau $P$ does not act freely on $\widetilde{\mathcal{A}}$, the isotropy group is the same $Z(G)$ (the center of $G$ ) for all $A \in \widetilde{\mathcal{A}}$, and $\widetilde{\mathcal{A}} / \mathrm{Gau} P$ is a differential manifold. If we define the group $\widetilde{\mathcal{G}}=\mathrm{Gau} P / Z(G)$, then $\widetilde{\mathcal{G}}$ acts freely on $\widetilde{\mathcal{A}}$ and $\widetilde{\mathcal{A}} \rightarrow \widetilde{\mathcal{A}} / \widetilde{\mathcal{G}}$ is a principal $\widetilde{\mathcal{G}}$-bundle (see, for example, [13] for details). In the preceding section, we have constructed a Gau $P$-equivariant prequantization bundle $\mathcal{U}_{c} \rightarrow \mathcal{A}$. If we restrict it to $\tilde{\mathcal{U}}_{c}=\widetilde{\mathcal{A}} \times U(1) \rightarrow \widetilde{\mathcal{A}}$, we hope that it will define a prequantization bundle over $\widetilde{\mathcal{A}} / \widetilde{\mathcal{G}}$, but there is a problem: the action of $Z(G)$ on $\widetilde{\mathcal{U}}_{c}$ does not need to be trivial and $\widetilde{\mathcal{G}}$ does not act on $\tilde{\mathcal{U}}_{c}$. Or, in an equivalent way, $\tilde{\mathcal{U}}_{c} / \mathrm{Gau} P \rightarrow \widetilde{\mathcal{A}} / \mathrm{Gau} P$ is not a $U(1)$-bundle. If the action of $Z(G)$ on $\mathcal{U}_{c}$ is trivial, then $\widetilde{\mathcal{G}}$ acts on $\mathcal{U}_{c}$, and restricting $\widetilde{\mathcal{A}}$ to $\tilde{\mathcal{U}}_{c} / \widetilde{\mathcal{G}} \rightarrow \widetilde{\mathcal{A}} / \widetilde{\mathcal{G}}$ we obtain a bundle over the moduli space of irreducible connections. This is the case for the trivial $S U$ (2)-bundle over a surface, as it is shown in [24]. If the action of $Z(G)$ on $\mathcal{U}_{c}$ is not trivial, we can define $\widetilde{G}=G / Z(G)$ and $\widetilde{P}=P / Z(G) \rightarrow M$, which is a principal $\widetilde{G}$-bundle. We also set $\widetilde{\mathbb{P}}=(P / Z(G)) \times \widetilde{\mathcal{A}}$ which is also a principal $\widetilde{G}$-bundle. The connection $\mathbb{A} \in \Omega^{1}(\mathbb{P}, \mathfrak{g})$ induces a connection $\widetilde{\mathbb{A}}$ on $\widetilde{\mathbb{P}}$ which is invariant under the action of $\widetilde{\mathcal{G}}$ (see [10] for details). The results of Sect. 6.2 can be applied to the bundle $\widetilde{P}=P / Z(G) \rightarrow M, N=\widetilde{\mathcal{A}}$ and the $\widetilde{\mathcal{G}}$-invariant connection $\widetilde{\mathbb{A}}$ on $\widetilde{\mathbb{P}}$, and we obtain a result analogous to Theorem 29 , but we should take polynomials and characteristic classes of $\widetilde{G}$ in place of $G$. If $(p, \Upsilon) \in \mathcal{I}_{\mathbb{Z}}^{r}(\tilde{G})$ and $c: C \rightarrow M$ with $\operatorname{dim} C=2 r-2$, we obtain $\varpi_{c} \in \Omega_{\widetilde{\mathcal{G}}}^{2}(\widetilde{\mathcal{A}})$ and a $\widetilde{\mathcal{G}}$-equivariant prequantization bundle $\left(\widetilde{\Xi}_{c} \widetilde{\mathcal{U}}_{c}\right)$ of $\left(\widetilde{\mathcal{A}}, \varpi_{c}\right)$, and taking the quotient a $U(1)$-bundle $\tilde{\mathcal{U}}_{c} / \widetilde{\mathcal{G}} \rightarrow \widetilde{\mathcal{A}} / \widetilde{\mathcal{G}}$. 
We consider only one example. If $G=S U(2)$, then $\widetilde{G}=S O(3)$. Both groups have the same Lie algebra $\mathfrak{s u}(2) \simeq \mathfrak{s o}(3)$. As they are connected, they have the same Weil polynomials $I(S U(2))=I(S O(3))$, but $I_{\mathbb{Z}}(S O(3)) \varsubsetneqq I_{\mathbb{Z}}(S U(2))$. For example, the second Chern polynomial $c_{2} \notin I_{\mathbb{Z}}(S O(3))$, but the first Pontryagin polynomial $p_{1}=4 c_{2} \in I_{\mathbb{Z}}(S O(3))$ (see [12, Formula 4.11]). If $c: C \rightarrow M$ is a map with $C$ a closed surface, the presymplectic structure $\underline{\sigma}_{c}$ on the moduli space of irreducible flat connections determined by $c$ and the second Chern class may not be prequantizable. But $4 \cdot \underline{\sigma}_{c}$ is always prequantizable by the bundle associated with the first Pontryagin class.

Let $(p, \Upsilon) \in \mathcal{I}_{\mathbb{Z}}^{r}(\widetilde{G})$. If $\widetilde{\mathcal{F}} \subset \widetilde{\mathcal{A}}$ is the space of irreducible flat connections, for $r \geq 2$ we have $\widetilde{\mathcal{F}} \subset \mu_{c}^{-1}(0)$. In particular, the restriction to $\widetilde{\mathcal{F}} \times U(1)$ of the form $\Xi_{c}$ is $\mathcal{G}$-basic. $\Xi_{c}$ projects onto a connection on $\widetilde{\mathcal{F}} / \widetilde{\mathcal{G}} \times U(1) \rightarrow \widetilde{\mathcal{F}} / \widetilde{\mathcal{G}}$, and we obtain a prequantization bundle of $\left(\widetilde{\mathcal{F}} / \widetilde{\mathcal{G}}, \underline{\sigma}_{c}\right)$, where $\underline{\sigma}_{c}$ is obtained from $\varpi_{c}$ by presymplectic reduction. For $r=2$ and $C=M$ a closed oriented surface, we obtain $\left(\sigma_{M}\right)_{A}(a, b)=2 \int_{M} p(a, b),\left(\mu_{M}\right)_{A}(X)=$ $-2 \int_{M} p(X, F)$ and $\left(\rho_{M}\right)_{A}(a)=\int_{M} p\left(A-A_{0}, a\right)$, for $A \in \mathcal{A}, a, b \in T_{A} \mathcal{A} \simeq \Omega^{1}(M, \operatorname{ad} P)$ and $X \in$ Lie $\mathcal{G}$. If $p: \mathfrak{g} \times \mathfrak{g} \rightarrow \mathbb{R}$ is a non-degenerate bilinear form, then $\sigma_{M}$ is a symplectic form and the moment map can be identified with the curvature map $A \mapsto F$. Hence, they coincide with the symplectic structure and moment map defined in [3]. As commented in Remark 31 in this case, our bundle also coincides with that of [24], and the connection $\Xi_{M}$ projects onto a connection on the quotient bundle $(\widetilde{\mathcal{F}} \times U(1)) / \widetilde{\mathcal{G}} \rightarrow \widetilde{\mathcal{F}} / \widetilde{\mathcal{G}}$. If $J$ is a complex structure on $M$, it induces a complex structure on $\mathcal{A}$ and $\sigma_{M}$ is of type $(1,1)$. As $\nabla \underline{\Xi}_{M}$ is a unitary connection, we conclude (see [13]) that it determines a holomorphic structure on $\underline{\mathcal{L}}_{M} \rightarrow \tilde{\mathcal{F}} / \widetilde{\mathcal{G}}$. We have similar results when $\operatorname{dim} M>2$ and $c: C \rightarrow M$ is a map with $\operatorname{dim} C=2$. If $c=\partial u$, the restriction of $S_{u}$ to $\widetilde{\mathcal{F}}$ is a $\Xi_{c}$-parallel section as it satisfies $\nabla \Xi_{c} S_{u}=0$ because $\left(\sigma_{u}\right)_{A}(a)=2 \int_{u} p(a, F)=0$ if $A \in \mathcal{F}$.

If $r \geq 3$ we have $\sigma_{c} \mid \mathcal{F}=0$, and in this case $\underline{\Xi}_{c}$ is a flat connection, and hence, its holonomy defines a cohomology class in $H^{1}(\widetilde{\mathcal{F}} / \widetilde{\mathcal{G}}, \mathbb{R} / \mathbb{Z}$ ) (see [10] for a generalization of this result to arbitrary dimensions).

\subsection{The action of automorphisms}

Let $\mathrm{Aut}^{+} P$ be the group of automorphisms preserving the orientation on $M$, and assume that $\mathcal{G}$ is a group acting on $P$ by elements of Aut $^{+} P$. In this case, we cannot choose $c: C \rightarrow M$ an arbitrary map because it should be $\mathcal{G}$ invariant. We only consider the cases $C=M$ (if $\partial M=0)$ and $C=\partial M$.

\subsubsection{Base manifold closed}

When $M$ is a closed manifold of dimension $2 r-2$, we can take $C=M$ and $c=\mathrm{id}_{M}$, which clearly is $\mathcal{G}$-invariant. As a consequence of Theorem 22, we obtain the following

Theorem 33 Let $P \rightarrow M$ principal $G$-bundle with $M$ closed, oriented and $\operatorname{dim} M=2 r-2$, $(p, \Upsilon) \in \mathcal{I}_{\mathbb{Z}}^{r}(G), A_{0}$ a background connection on $P$ and a group $\mathcal{G}$ acting on $P$ by elements of Aut $^{+} P$. These data determine an action of $\mathcal{G}$ on $\mathcal{U}_{M}=\mathcal{A} \times U(1) \rightarrow \mathcal{A}$ by $U(1)$-bundle automorphisms such that the connection $\Xi_{M}=\theta-2 \pi i \rho_{M}$ is $\mathcal{G}$-invariant and the equivariant curvature of $\Xi_{M}$ is $\varpi_{M}$.

Theorem 33 extends to arbitrary bundles the results of [1,2] for trivial bundles over a surface. 


\subsubsection{Base manifold with boundary}

Now we assume that $M$ is a compact oriented manifold of dimension $2 r-1$ with boundary $\partial M$. We chose $C=\partial M$ and $c=\operatorname{id}_{M}$. By applying Theorem 22, we obtain the following

Theorem 34 Let $P \rightarrow M$ principal $G$-bundle with $M$ compact and oriented with boundary $\partial M$ and $\operatorname{dim} M=2 r-1$. If a group $\mathcal{G}$ acts on $P$ by elements of $\operatorname{Aut}^{+} P,(p, \Upsilon) \in \mathcal{I}_{\mathbb{Z}}^{r}(G)$ and $A_{0}$ is a background connection on $P$, these data determine an action of $\mathcal{G}$ on $\mathcal{U}_{\partial M}=$ $\mathcal{A} \times U(1) \rightarrow \mathcal{A}$ by $U(1)$-bundle automorphisms such that the connection $\Xi_{\partial M}=\theta-2 \pi i \rho_{\partial M}$ is $\mathcal{G}$-invariant and the equivariant curvature of $\Xi_{\partial M}$ is $\varpi_{\partial M}$.

Furthermore, $S_{M}=\exp \left(-2 \pi i \cdot \int_{M} T p\left(A, A_{0}\right)\right)$ determines $\mathcal{G}$-equivariant section of $\mathcal{U}_{\partial M} \rightarrow \mathcal{A}$, and we have $\nabla^{\Xi_{\partial M}} S_{M}=-2 \pi i \sigma_{M} \cdot S_{M}$.

\section{Riemannian metrics and diffeomorphisms}

In this section, we apply our results to the space of Riemannian metrics and the action of diffeomorphisms. One possible approach to do this is to apply the results of Sect. 6 to the structures on the space of metrics defined in [16-18]. However, we follow a different approach: we obtain the prequantization bundle by pulling back the bundles on the space of connections using the Levi-Civita map.

If $M$ is an oriented manifold and we take $P=F M$, the group $\mathcal{G}=\operatorname{Diff}^{+} M$ of orientation-preserving diffeomorphisms acts on $F M$ by automorphisms. The Levi-Civita map LC: $\mathfrak{M e t} M \rightarrow \mathcal{A}$ which assigns to a Riemannian metric $g$ its Levi-Civita connection $L C(g)=\omega^{g}$ is $\mathcal{G}$-equivariant. If we denote by $p_{k} \in I_{\mathbb{Z}}^{2 k}(G L(n, \mathbb{R}))$ the $k$-th Pontryagin polynomial and by $\Upsilon_{k} \in H^{4 k}(\mathbf{B} G L(n, \mathbb{R})) \simeq H^{4 k}(\mathbf{B} O(n))$ the $k$-th Pontryagin class, then $p_{k}$ and $\Upsilon_{k}$ are compatible. We fix a polynomial $p \in \mathbb{Z}\left[p_{1}, \ldots, p_{n / 2}\right] \subset I_{\mathbb{Z}}^{\bullet}(G L(n, \mathbb{R}))$ of degree $2 r$ and the corresponding characteristic class $\Upsilon \in H^{4 k}(\mathbf{B} G L(n, \mathbb{R}))$.

\subsection{Closed manifolds}

Let $M$ be a compact closed manifold of dimension $4 r-2$. If we fix a background connection $A_{0}$ on $F M$, we can apply the results of Sect. 7.3.1, and we obtain a $\mathcal{G}$-equivariant prequantization bundle $\left(\mathcal{U}_{M}, \Xi_{M}\right)$ of the equivariant form $\varpi_{M}=\sigma_{M}+\mu_{M} \in \Omega_{\mathcal{G}}^{2}(\mathcal{A})$. Using the Levi-Civita map, we obtain $\mathcal{U}_{M}^{\prime}=\mathrm{LC}^{*} \mathcal{U}_{M}, \Xi_{M}^{\prime}=\mathrm{LC}^{*} \Xi_{M}$, and $\left(\mathcal{U}_{M}^{\prime}, \Xi_{M}^{\prime}\right)$ is a $\mathcal{G}$-equivariant prequantization bundle of $\varpi_{M}^{\prime}=\mathrm{LC}^{*} \varpi_{M} \in \Omega_{\mathcal{G}}^{2}(\operatorname{Met} M)$. It can be seen that $\varpi_{M}^{\prime}=\sigma_{M}^{\prime}+\mu_{M}^{\prime}$ coincide with the presymplectic structure and moment map defined in [18]. We study in detail the simplest case.

\subsubsection{Dimension 2}

Let $M$ be a closed surface and $p_{1}(X)=-\frac{1}{8 \pi^{2}} \operatorname{tr}\left(X^{2}\right)$ is the first Pontryagin polynomial. The presymplectic reduction of ( Met $M, \varpi)$ is studied in [18], and the result is that $\left(\mu_{M}^{\prime}\right)^{-1}(0)=$ $\mathfrak{M e t}^{*} M$ is the space of metrics of constant curvature.

If $M$ has genus $\gamma>1$ and $\mathfrak{M e t}_{-1} M$ is the space of metrics of constant curvature -1 , we have $\mathfrak{M e t}_{-1} M \subset \mu^{-1}(0)$. The connected component with the identity Diff ${ }_{0} M$ acts freely on $\mathfrak{M e t}_{-1} M$ and the Teichmüller space of $M$ is defined by $\mathcal{T}(M)=\mathfrak{M e t}_{-1} M / \operatorname{Diff}_{0} M$, which, as it is well known (e.g., see [26]), is a manifold of real dimension $6 \gamma-6$. It is proved in [18] that the form obtained from $\sigma_{M}^{\prime}$ by symplectic reduction is $\underline{\sigma}_{M}^{\prime}=\frac{1}{2 \pi^{2}} \sigma_{\mathrm{WP}}$, where $\sigma_{\mathrm{WP}}$ 
is the symplectic form of the Weil-Petersson metric on $\mathcal{T}(M)$. We define the quotient bundle $\mathcal{W}_{M}=\left(\mathfrak{M e t}_{-1} M \times U(1)\right) / \operatorname{Diff}_{0} M \rightarrow \mathcal{T}(M)$. As $\mathfrak{M e t}_{-1} M \subset\left(\mu_{M}^{\prime}\right)^{-1}(0)$, the connection $\Xi_{M}^{\prime}$ is projectable onto a connection $\vartheta_{M}$ on $\mathcal{W}_{M}$. Moreover, as $\underline{\sigma}_{M}^{\prime}$ is of type $(1,1)$ and $\vartheta_{M}$ is a unitary connection, we conclude (e.g., see [13]) that $\nabla^{\vartheta_{M}}$ determines a holomorphic structure on the line bundle $\mathcal{L}_{M} \rightarrow \mathcal{T}(M)$ associated with $\mathcal{W}_{M}$.

Furthermore, the first Pontryagin class determines the action on $\mathcal{L}_{M}$ of the elements of Diff $^{+} M$ not connected with the identity and hence an action of $\Gamma_{M}=\operatorname{Diff}^{+} M / \operatorname{Diff}_{0} M$ (the mapping class group of $M)$ on $\mathcal{L}_{M}$ which preserves $\nabla^{\vartheta_{M}}$. We conclude that $\left(\mathcal{L}_{M}, \nabla^{\vartheta_{M}}\right)$ is a $\Gamma_{M}$-equivariant holomorphic Hermitian prequantization bundle for $\left(\mathcal{T}(M), \frac{1}{2 \pi^{2}} \sigma_{\mathrm{WP}}\right)$.

Similar prequantization bundles are constructed, for example, in [21] and in [27] by different techniques. We note that our construction is not specific of two dimensions and can be applied to any manifold of dimension $4 r-2$.

\subsection{Manifolds with boundary}

If $M$ is a compact manifold of dimension $4 r-1$ with boundary, we can apply the results of Sect. 7.3.2 and we obtain a $\mathcal{G}$-equivariant prequantization bundle $\left(\mathcal{U}_{\partial M}, \Xi_{\partial M}\right)$ of $\varpi_{\partial M}=$ $\sigma_{\partial M}+\mu_{\partial M} \in \Omega_{\mathcal{G}}^{2}(\mathcal{A})$. By using the Levi-Civita map, we obtain $\mathcal{U}_{\partial M}^{\prime}=\mathrm{LC}^{*} \mathcal{U}_{\partial M}, \Xi_{\partial M}^{\prime}=$ $\mathrm{LC}^{*} \Xi_{\partial M}$ and $\left(\mathcal{U}_{\partial M}^{\prime}, \Xi_{\partial M}^{\prime}\right)$ is a $\mathcal{G}$-equivariant prequantization bundle of $\varpi_{\partial M}^{\prime}=\mathrm{LC}^{*} \varpi_{\partial M} \in$ $\Omega_{\mathcal{G}}^{2}(\operatorname{Met} M)$. Furthermore, we have the following

Theorem 35 If $M$ is a compact oriented manifold with boundary $\partial M$ then $S(g)=$ $\exp \left(-2 \pi i \cdot \int_{M} T p\left(\omega^{g}, A_{0}\right)\right)$ determines $\mathcal{G}$-invariant section of $\mathcal{U}_{\partial M}^{\prime} \rightarrow \mathfrak{M e t} M$.

Hence, we have found a Chern-Simons line for Riemannian metrics.

We note that the prequantization bundle on $\mathcal{T}(M)$ is defined in [21] by using a similar Chern-Simons line in dimension 3. They express the surface as the boundary of a 3-manifold, and they use a definition of the bundle similar to that in [24] for connections. As in the case of connections, this procedure cannot be extended to higher dimensions.

Acknowledgements The research was supported by "Proyecto de investigación Santander-UCM PR26/1620305".

\section{References}

1. Andersen, J.E.: The Witten-Reshetikhin-Turaev invariants of finite order mapping tori I. J. Reine Angew. Math. 681, 1-38 (2013)

2. Andersen, J.E., Himpel, B., Jørgensen, S.F., Martens, J., McLelland, B.: The Witten-Reshetikhin-Turaev invariant for links in finite order mapping tori I. Adv. Math. 304, 131-178 (2017)

3. Atiyah, M.F., Bott, R.: The Yang-Mills equations over Riemann surfaces. Phil. Trans. R. Soc. Lond. A 308, 523-615 (1982)

4. Atiyah, M.F., Singer, I.: Dirac operators coupled to vector potentials. Proc. Natl. Acad. Sci. USA 81, 2597-2600 (1984)

5. Bär, C., Becker, C.: Differential Characters. Lecture Notes in Mathematics, vol. 2112. Springer, Berlin (2014)

6. Berline, N., Vergne, M.: Classes caractéristiques équivariantes. Formules de localisation en cohomologie équivariante. C. R. Acad. Sci. Paris 295, 539-541 (1982)

7. Berline, N., Getzler, E., Vergne, M.: Heat Kernels and Dirac Operators. Springer, Berlin (1992)

8. Bonora, L., Cotta-Ramusino, P., Rinaldi, M., Stasheff, J.: The evaluation map in field theory, sigma-models and strings-II. Commun. Math. Phys. 114, 381-438 (1988)

9. Bott, R., Tu, L.: Equivariant Characteristic Classes in the Cartan Model, Geometry, Analysis and Applications (Varanasi, 2000). World Science Publishing, River Edge, pp. 3-20 (2001) 
10. Castrillón López, M., Ferreiro Pérez, R.: Differential characters and cohomology of the moduli of flat connections. arXiv:1711.06995

11. Cheeger, J., Simons, J.: Differential characters and geometric invariants. In: Geometry and Topology, Proc. Spec. Year, College Park/MD 1983/84, Lecture Notes in Mathematics, vol. 1167. Springer, Berlin (1985)

12. Dijkgraaf, R., Witten, E.: Topological gauge theories and group cohomology. Commun. Math. Phys. 129, 393-429 (1990)

13. Donaldson, S.K., Kronheimer, P.B.: The Geometry of Four-Manifolds. Oxford University Press, Oxford (1990)

14. Dupont, J.L., Johansen, F.L.: Remarks on determinant line bundles, Chern-Simons forms and invariants. Math. Scand. 91, 5-26 (2001)

15. Ferreiro Pérez, R.: Equivariant characteristic forms in the bundle of connections. J. Geom. Phys. 54, 197-212 (2005)

16. Ferreiro Pérez, R.: Local anomalies and local equivariant cohomology. Commun. Math. Phys. 286, 445458 (2009)

17. Ferreiro Pérez, R., Muñoz Masqué, J.: Natural connections on the bundle of Riemannian metrics. Monatsh. Math. 155, 67-78 (2008)

18. Ferreiro Pérez, R., Muñoz Masqué, J.: Pontryagin forms on $(4 k-2)$-manifolds and symplectic structures on the spaces of Riemannian metrics. Differ. Geom. Appl. 30, 206-215 (2012)

19. Freed, D.S.: Classical Chern-Simons theory, part 1. Adv. Math. 113, 237-303 (1995)

20. Freed, D.S.: Classical Chern-Simons theory, part 2. Houst. J. Math. 28, 293-310 (2002)

21. Guillarmou, C., Moroianu, S.: Chern-Simons line bundle on Teichmüller space. Geom. Topol. 18(1), 327-377 (2014)

22. Guillemin, V., Sternberg, S.: Supersymmetry and Equivariant de Rham Theory. Springer, Berlin (1999)

23. May, J.P.: A Concise Course in Algebraic Topology, Chicago Lectures in Mathematics (1999)

24. Ramadas, T.R., Singer, I.M., Weitsman, J.: Some comments on Chern-Simons gauge theory. Commun. Math. Phys. 126, 409-420 (1989)

25. Salamon, D.A.: Notes on flat connections and the loop group. University of Warwick, Preprint (1998)

26. Tromba, A.J.: Teichmüller Theory in Riemannian Geometry. Birkhäuser, Basel (1992)

27. Wolpert, S.A.: On obtaining a positive line bundle from the Weil-Petersson class. Am. J. Math. 107, 1485-1507 (1985) 